\title{
COMPARISON OF HIGH-ACCURACY FINITE-DIFFERENCE METHODS FOR LINEAR WAVE PROPAGATION*
}

\author{
DAVID W. ZINGG ${ }^{\dagger}$
}

\begin{abstract}
This paper analyzes a number of high-order and optimized finite-difference methods for numerically simulating the propagation and scattering of linear waves, such as electromagnetic, acoustic, and elastic waves. The spatial operators analyzed include compact schemes, noncompact schemes, schemes on staggered grids, and schemes which are optimized to produce specific characteristics. The time-marching methods include Runge-Kutta methods, Adams-Bashforth methods, and the leapfrog method. In addition, the following fully-discrete finite-difference methods are studied: a one-step implicit scheme with a three-point spatial stencil, a one-step explicit scheme with a five-point spatial stencil, and a two-step explicit scheme with a five-point spatial stencil. For each method, the number of grid points per wavelength required for accurate simulation of wave propagation over large distances is presented. The results provide a clear understanding of the relative merits of the methods compared, especially the trade-offs associated with the use of optimized methods. A numerical example is given which shows that the benefits of an optimized scheme can be small if the waveform has broad spectral content.
\end{abstract}

Key words. finite-difference methods, wave propagation, electromagnetics, acoustics, Maxwell equations

AMS subject classifications. 65M05, 78A40, 78M20, 76Q05

PII. S1064827599350320

Introduction. Numerical simulation can play an important role in the context of engineering design and in improving our understanding of complex systems. The simulation of wave phenomena, including electromagnetic, elastic, and acoustic waves, is an area of active research. Lighthill [24] and Taflove [40] discuss the prospects for computational aeroacoustics and electromagnetics, respectively. The computational requirements for accurate simulations of the propagation and scattering of waves can be high, particularly if the size of the geometry under study is much larger than the wavelength. Consequently, there has been considerable recent effort directed towards improving the efficiency of numerical methods for simulating wave phenomena.

In electromagnetics, the most popular approach to the numerical solution of the time-domain Maxwell equations for numerous applications has been the algorithm of Yee [50], which was named the finite-difference time-domain (FDTD) method by Taflove [41]. This algorithm combines second-order centered differences on a staggered grid in space with second-order staggered leapfrog time marching. Its main attributes are its very low cost per grid node and lack of dissipative error. Yee's method is often applied using Cartesian grids, with a special treatment of curved boundaries [20]. Extension to curvilinear grids was carried out by Fusco [11]. Madsen and Ziolowski [28] put the method into a finite-volume framework applicable to unstructured grids. Vinokur and Yarrow $[47,48]$ developed a related finite-surface method with advantages at boundaries and grid singularities.

Other methods which have been successfully applied to the time-domain Maxwell equations include the upwind Lax-Wendroff approach used by Shankar, Mohamma-

\footnotetext{
* Received by the editors January 28, 1999; accepted for publication(in revised form) December 10, 1999; published electronically July 13, 2000.

http://www.siam.org/journals/sisc/22-2/35032.html

${ }^{\dagger}$ University of Toronto Institute for Aerospace Studies, 4925 Dufferin St., Downsview, Ontario, Canada M3H 5T6 (dwz@oddjob.utias.utoronto.ca).
} 
dian, and Hall [38], the characteristic-based fractional-step method of Shang [36], and the finite-element method of Cangellaris, Lin, and Mei [5]. Although all of these second-order methods have been used with a great deal of success, they are efficient only for geometries of moderate electrical size, on the order of 20 wavelengths or less. For wave propagation over longer distances, the grid resolution requirements of second-order methods can become excessive, leading to impractical CPU and memory requirements. This has motivated the development of higher-order methods which produce smaller errors for a given grid resolution, such as the extension of Yee's method to fourth-order in space [40] and the methods of Liu [25], Shang and Gaitonde [37], Zingg et al. [52, 55, 18, 19], Petropoulos [31], Turkel and Yefet [44], and Young, Gaitonde, and Shang [51], many of which use staggered grids.

In seismology, the need for higher-order methods has been recognized for some time. Alford, Kelly, and Boore [2], Marfurt [29], Dablain [9], and Sei [34] present higher-order algorithms for the elastic wave equation. Similarly, higher-order finitedifference methods have been developed for acoustic applications by Gottlieb and Turkel [13], Cohen and Joly [8], and Davis [10], for example.

It can be advantageous to modify the coefficients of a potentially higher-order method, thereby lowering the order of accuracy, to produce reduced errors over a range of wavenumbers. We refer to such schemes as optimized schemes [52]. This approach was first proposed by Vichnevetsky and De Schutter [45] and later studied in more detail by Holberg [16] and Lele [23]. Haras and Ta'asan [15] and later Kim and Lee [21] further refined the optimization technique used by Lele. The papers by Holberg and Lele spawned a number of optimized schemes, including those presented by $\mathrm{Hu}$, Hussaini, and Manthey [17], Lockard, Brentner, and Atkins [27], Sguazzero, Kindelan, and Kamel [35], Tam [42], Tam and Webb [43], Zingg and Lomax [53], and Zingg, Lomax, and Jurgens [52, 55]. See Wells and Renaut [49] for a discussion of several of these schemes in the context of computational aeroacoustics.

In addition to the accuracy of the interior differencing scheme, there are several other important issues in simulating wave phenomena. High-order and optimized methods often have a large spatial stencil which cannot be used near boundaries. This necessitates the use of numerical boundary schemes which must be suitably accurate relative to the interior scheme [14] and stable. These can be difficult to obtain, and this represents a significant obstacle to the use of higher-order methods. Recent progress is reported by Olsson [30] and Carpenter, Gottlieb, and Abarbanel [6, 7]. Zingg and Lomax [54] present a fifth-order numerical boundary scheme which produces a stable scheme in conjunction with sixth-order centered differences. An improved version, which is stable for curvilinear grids, is given by Jurgens and Zingg [19]. Another important consideration is the boundary condition at the outer boundary of the domain, which inevitably causes spurious reflection. As a result of recent developments in this area, such as perfectly matched absorbing layers [1, 4, 12, 32, 57], such reflections can be substantially reduced. Consequently, the numerical errors introduced by the interior differencing scheme are often dominant, and the choice of the interior scheme is critical to the efficient simulation of wave phenomena.

An efficient discretization of a partial differential equation governing a linear wave phenomenon reliably maintains the numerical error below an acceptable threshold, which is problem dependent, at the lowest possible cost. The cost includes processing time and memory, although development cost can be considered as well. Numerical errors arise from both the spatial and the temporal discretization. They include both phase and amplitude errors, which depend on the wavenumber, the grid spacing, 
the Courant number, and the direction of propagation relative to the grid. The dependence of the phase speed on the wavenumber results in numerical dispersion [46], while the amplitude error results in numerical dissipation. The dependence of these errors on the direction of wave propagation relative to the grid leads to numerical anisotropy. Fourier analysis provides a straightforward means of calculating these errors $[46,26]$. Although this simplified analysis excludes errors associated with nonuniform grids and boundaries, it is a very useful tool for scheme evaluation and development. Good performance under the conditions of Fourier analysis, i.e., uniform grids and periodic boundary conditions, is a necessary condition for good performance under more general conditions.

Through Fourier analysis, one can calculate the phase and amplitude error of a given method as a function of the wavenumber. However, this information can be difficult to interpret. A more useful approach used by Lockard, Brentner, and Atkins [27] is to plot the grid resolution in terms of grid points per wavelength (PPW) required to maintain global phase and amplitude errors below a specified threshold as a function of the distance of propagation expressed in terms of the number of wavelengths traveled. This is not only an excellent metric for scheme development and comparison, but it also provides useful information for applying the methods to specific problems.

In this paper, we study and compare the grid resolution requirements of several high-order and optimized schemes, including spatial discretizations, time-marching methods, and combined time-space discretizations. Emphasis is on schemes requiring under $30 \mathrm{PPW}$ for accurate simulations with propagation distances of up to 200 wavelengths. The purpose is twofold. First, the results can aid in the evaluation and application of high-accuracy methods and are especially helpful in clarifying the behavior of optimized schemes. Second, the framework presented can be used in the assessment of new methods.

Finite-difference schemes compared. In this section, we present the various finite-difference schemes in the context of the linear advection equation given by

$$
\frac{\partial u}{\partial t}+a \frac{\partial u}{\partial x}=0
$$

where $u$ is a scalar quantity propagating with speed $a$, which is real and positive. The schemes under study can be divided into two distinct groups. In the first group, the spatial and temporal discretizations are independent, i.e., a discretization is applied to the spatial derivative to produce a system of ordinary differential equations (ODEs), which is solved numerically using a time-marching method. Hence we first study spatial discretizations and time-marching methods separately and later consider specific combinations. The spatial difference operators are approximations to $\partial / \partial x$. The time-marching methods are presented as applied to a scalar ODE of the form

$$
\frac{d u}{d t}=f(u, t)
$$

The second group of methods involves the simultaneous discretization of space and time; there is no intermediate semidiscrete form. These are presented as applied to (1) itself. The coefficients of all of the schemes studied are given in the appendix.

The methods can also be classified as dissipative or nondissipative. A spatial discretization is nondissipative, i.e., produces no amplitude error, if it is skew symmetric. When combined with certain time-marching methods, such as the leapfrog 
method, the resulting full discretization is nondissipative, that is, the amplitude of the solution will neither grow nor decay except in the presence of boundaries. Combined space-time discretizations can be nondissipative as well. This is generally a useful property in that it is consistent with the physics in preserving certain energy norms. Thus nondissipative schemes are widely used with great success, especially in solving the Maxwell equations. However, dissipative schemes can be effective as well, as long as the numerical dissipation is carefully controlled. For example, in the scheme of Zingg, Lomax, and Jurgens [55], the dissipative error is smaller than the phase error for all wavenumbers. Therefore, any mode for which the numerical dissipation is excessive already suffers from excessive phase error. Dissipative schemes have the advantage that high-frequency spurious waves, arising from boundaries, for example, are damped [13]. Furthermore, dissipative schemes can, at least in principle, be applied to nonlinear problems.

Spatial difference operators. On a uniform grid with $x_{j}=j \Delta x$ and $u_{j}=$ $u\left(x_{j}\right)$, compact centered-difference schemes of up to tenth order can be represented by the following formula:

$$
\begin{array}{r}
\beta\left(\delta_{x} u\right)_{j-2}+\alpha\left(\delta_{x} u\right)_{j-1}+\left(\delta_{x} u\right)_{j}+\alpha\left(\delta_{x} u\right)_{j+1}+\beta\left(\delta_{x} u\right)_{j+2} \\
=\frac{1}{2 \Delta x}\left[\frac{c}{3}\left(u_{j+3}-u_{j-3}\right)+\frac{b}{2}\left(u_{j+2}-u_{j-2}\right)+a\left(u_{j+1}-u_{j-1}\right)\right],
\end{array}
$$

where $\left(\delta_{x} u\right)_{j}$ is an approximation to $\partial u / \partial x$ at node $j$. These schemes produce no amplitude error. Noncompact schemes of up to sixth order are obtained with $\beta=$ $\alpha=0$.

Haras and Ta'asan [15] revisited the compact schemes of Lele [23] based on (3) using a more sophisticated optimization procedure. They developed tridiagonal schemes $(\beta=0)$ and tridiagonal schemes with a five-point stencil $(\beta=c=0)$ as well. In each case, Haras and Ta'asan present several methods which result from different parameters in the optimization procedure. In the comparisons below, we will include only those schemes which are best according to our criterion, i.e., those schemes which require the fewest grid points per wavelength for accurate wave propagation over a distance of 200 wavelengths.

The spatial operator of Zingg, Lomax, and Jurgens [52, 55] is noncompact with a seven-point stencil. The operator is divided into a skew-symmetric part given by

$$
\left(\delta_{x}^{a} u\right)_{j}=\frac{1}{\Delta x}\left[a_{3}\left(u_{j+3}-u_{j-3}\right)+a_{2}\left(u_{j+2}-u_{j-2}\right)+a_{1}\left(u_{j+1}-u_{j-1}\right)\right]
$$

and a symmetric part given by

(5) $\left(\delta_{x}^{s} u\right)_{j}=\frac{1}{\Delta x}\left[d_{3}\left(u_{j+3}+u_{j-3}\right)+d_{2}\left(u_{j+2}+u_{j-2}\right)+d_{1}\left(u_{j+1}+u_{j-1}\right)+d_{0} u_{j}\right]$.

The symmetric part provides dissipation of spurious high-wavenumber components of the solution. The magnitude of the dissipative component is chosen such that the amplitude error produced is less than the phase error. [52, 55] include a maximum-order scheme and an optimized scheme. The optimized scheme was designed to minimize the maximum phase and amplitude errors for waves resolved with at least $10 \mathrm{PPW}$. It is superior for distances of travel of up to 330 wavelengths. Hence we do not consider the maximum-order scheme here. Tam and Webb [43] have also developed an optimized scheme based on the skew-symmetric operator given in (4). 
Lockard, Brentner, and Atkins [27] present an optimized upwind-biased noncompact spatial difference operator based on an eight-point stencil. It can be written in the form

$$
\left(\delta_{x} u\right)_{j}=\frac{1}{\Delta x} \sum_{m=-4}^{3} a_{m} u_{j+m} .
$$

The operator is optimized for waves resolved with at least 7 PPW and provides excellent accuracy up to about 340 wavelengths of travel. Note that this operator can also be written as the sum of a skew-symmetric and a symmetric operator. A nine-point stencil is required for systems of equations with wave speeds of opposite sign.

Differencing schemes on staggered grids are well suited to problems in which the time derivative of one variable depends on the spatial derivative of the other, and viceversa. This is the case for the time-domain Maxwell equations or the Euler equations linearized about a reference state with zero velocity, for example. Centered staggered differencing schemes of up to sixth order can be written in the form

$$
\left(\delta_{x} u\right)_{j}=\frac{1}{\Delta x}\left[b_{1}\left(u_{j+1 / 2}-u_{j-1 / 2}\right)+b_{2}\left(u_{j+3 / 2}-u_{j-3 / 2}\right)+b_{3}\left(u_{j+5 / 2}-u_{j-5 / 2}\right)\right] \text {. }
$$

Time-marching methods. There are many considerations involved in selecting a time-marching method, including efficiency, i.e., accuracy per unit computational effort, stability, and memory use. Since wave propagation problems are generally not particularly stiff, explicit methods are appropriate. Thus the Adams-Bashforth and Runge-Kutta families are suitable candidates. Although other methods, such as predictor-corrector methods, can be used, we analyze only a few popular options, as well as some recently introduced methods.

Since a large portion of the computational effort is generally associated with evaluation of the derivative function, one can approximately assess the efficiency of a time-marching method by accounting for the number of derivative function evaluations per time step. Runge-Kutta methods require one derivative function evaluation per stage. Zingg and Chisholm [56] have shown that for linear ODEs with constant coefficients, Runge-Kutta methods of up to sixth order can be derived with a number of stages equal to the order. However, the memory requirements of the methods of orders five and six are high. For nonlinear ODEs and linear ODEs with nonconstant coefficients, six stages are required for fifth-order accuracy and seven stages for sixthorder accuracy [22].

An alternative approach is to use low-storage multistage methods which are highorder for homogeneous linear ODEs but second-order otherwise [55]. Haras and Ta'asan [15] and Zingg, Lomax, and Jurgens [52, 55] present five- and six-stage methods of this type, respectively. For example, when applied to (2), the six-stage method of Zingg, Lomax, and Jurgens [52, 55] is given by

$$
\begin{aligned}
& u_{n+\alpha_{1}}^{(1)}=u_{n}+h \alpha_{1} f_{n}, \\
& u_{n+\alpha_{2}}^{(2)}=u_{n}+h \alpha_{2} f_{n+\alpha_{1}}^{(1)}, \\
& u_{n+\alpha_{3}}^{(3)}=u_{n}+h \alpha_{3} f_{n+\alpha_{2}}^{(2)}, \\
& u_{n+\alpha_{4}}^{(4)}=u_{n}+h \alpha_{4} f_{n+\alpha_{3}}^{(3)},
\end{aligned}
$$




$$
\begin{aligned}
u_{n+\alpha_{5}}^{(5)} & =u_{n}+h \alpha_{5} f_{n+\alpha_{4}}^{(4)}, \\
u_{n+1} & =u_{n}+h f_{n+\alpha_{5}}^{(5)},
\end{aligned}
$$

where $h=\Delta t$ is the time step, $t_{n}=n h, u_{n}=u\left(t_{n}\right)$, and

$$
f_{n+\alpha}^{(k)}=f\left(u_{n+\alpha}^{(k)}, t_{n}+\alpha h\right) .
$$

The five-stage method of [15] is analogous. In their maximum-order form (for homogeneous ODEs), these methods are not stable for pure central (skew-symmetric) differencing in space. Consequently, Haras and Ta'asan modify the coefficients of the five-stage scheme to produce stability, while Zingg, Lomax, and Jurgens [52, 55] add a dissipative (symmetric) component to their spatial operator, as given in (5).

Adams-Bashforth methods require only one derivative function evaluation per time step, and thus can be more efficient than Runge-Kutta methods of equal order. Since they are multistep methods, however, they require extra storage. Furthermore, Adams-Bashforth methods of order higher than four have extremely restrictive stability bounds. Thus the time-marching methods we consider here are the fourth-order Adams-Bashforth method, the low-storage fourth-order Runge-Kutta method for linear ODEs given by Zingg and Chisholm [56], and the low-storage five- and six-stage methods described above. In terms of stability and Fourier error analysis, the fourthorder Runge-Kutta method of [56] is identical to the classical fourth-order RungeKutta method. With respect to memory requirements, the fourth-order AdamsBashforth method requires five memory locations per dependent variable, while the other methods considered require only two.

A natural choice of time-marching method for use with staggered spatial differencing is the second-order staggered leapfrog method, given by

$$
u_{n+1}=u_{n}+h f_{n+1 / 2} .
$$

When used with a nondissipative spatial scheme, as in the FDTD method, the resulting fully-discrete operator produces no amplitude error. Furthermore, the staggered leapfrog method generally produces a leading phase error, which can offset the phase lag usually produced by centered spatial differences. At specific Courant numbers and angles of propagation, the perfect-shift property ${ }^{1}$ can be obtained, leading to exact propagation for all wavenumbers. Although this has little practical significance, fully-discrete methods which possess this property are generally most accurate when used near the perfect-shift conditions.

Simultaneous space-time discretizations. The scheme of Davis [10] is a nondissipative implicit scheme with a three-point spatial stencil. When applied to the linear advection equation, it is given by

$$
a_{0} u_{j}^{n+1}+a_{1} u_{j-1}^{n+1}+a_{2} u_{j+1}^{n+1}=b_{0} u_{j}^{n}+b_{1} u_{j-1}^{n}+b_{2} u_{j+1}^{n}
$$

with $a_{0}=b_{0}, a_{1}=b_{2}$, and $a_{2}=b_{1}$, where $u_{j}^{n}=u\left(x_{j}, t_{n}\right)$. Being implicit, this scheme requires more computational expense than explicit schemes. However, it achieves fourth-order accuracy in time and space with a three-point spatial stencil.

The scheme of Gottlieb and Turkel [13] is an extension of the Lax-Wendroff approach to fourth-order in space, remaining second-order in time. It is an explicit

\footnotetext{
${ }^{1}$ This refers to the situation when the error from the spatial discretization precisely cancels that from the temporal discretization.
} 
one-step scheme but requires a five-point stencil. When applied to the linear advection equation, it is given by

$$
\begin{aligned}
u_{j}^{(1)} & =u_{j}^{n}+\frac{a h}{6 \Delta x}\left(u_{j+2}^{n}-8 u_{j+1}^{n}+7 u_{j}^{n}\right), \\
u_{j}^{n+1} & =\frac{1}{2}\left(u_{j}^{n}+u_{j}^{(1)}\right)+\frac{a h}{12 \Delta x}\left(-u_{j-2}^{(1)}+8 u_{j-1}^{(1)}-7 u_{j}^{(1)}\right) .
\end{aligned}
$$

The two-stage form is motivated by nonlinear problems. This method is dissipative and has received considerable use in nonlinear problems. The justification for having a lower order of accuracy in time than in space is based on the idea that the system is at least mildly stiff. Hence the time-step restriction based on the parasitic modes, i.e., the stability limit, is often much more stringent than that for the driving modes, i.e., the accuracy limit. Note, however, that many wave propagation problems are not stiff at all.

It is straightforward to extend this approach to fourth-order in time without increasing the stencil. For example, one can derive the following dissipative one-step fourth-order method:

$$
u_{j}^{n+1}=u_{j}^{n}-a h\left(\delta_{x}^{(4)} u\right)_{j}^{n}+\frac{h^{2} a^{2}}{2}\left(\delta_{x x}^{(4)} u\right)_{j}^{n}-\frac{h^{3} a^{3}}{6}\left(\delta_{x x x}^{(2)} u\right)_{j}^{n}+\frac{h^{4} a^{4}}{24}\left(\delta_{x x x x}^{(2)} u\right)_{j}^{n},
$$

where $\delta_{x}^{(4)}$ is a fourth-order centered difference approximation to a first derivative, $\delta_{x x}^{(4)}$ is a fourth-order centered difference approximation to a second derivative, $\delta_{x x x}^{(2)}$ is a second-order centered difference approximation to a third derivative, and $\delta_{x x x x}^{(2)}$ is a second-order centered approximation to a fourth derivative. All of the operators on the right-hand side require a five-point stencil, as follows:

$$
\begin{aligned}
\left(\delta_{x}^{(4)} u\right)_{j} & =\frac{1}{12 \Delta x}\left(u_{j-2}-8 u_{j-1}+8 u_{j+1}-u_{j+2}\right), \\
\left(\delta_{x x}^{(4)} u\right)_{j} & =\frac{1}{12 \Delta x^{2}}\left(-u_{j-2}+16 u_{j-1}-30 u_{j}+16 u_{j+1}-u_{j+2}\right), \\
\left(\delta_{x x x}^{(2)} u\right)_{j} & =\frac{1}{2 \Delta x^{3}}\left(-u_{j-2}+2 u_{j-1}-2 u_{j+1}+u_{j+2}\right), \\
\left(\delta_{x x x x}^{(2)} u\right)_{j} & =\frac{1}{\Delta x^{4}}\left(u_{j-2}-4 u_{j-1}+6 u_{j}-4 u_{j+1}+u_{j+2}\right) .
\end{aligned}
$$

This scheme is quite complicated to apply in multidimensions. Hence we will consider a simpler scheme which is fourth-order in time and space, the following nondissipative two-step explicit scheme:

$$
u_{j}^{n+1}=u_{j}^{n-1}-2 a h\left(\delta_{x}^{(4)} u\right)_{j}^{n}-\frac{h^{3} a^{3}}{3}\left(\delta_{x x x}^{(2)} u\right)_{j}^{n},
$$

where $\delta_{x}^{(4)}$ and $\delta_{x x x}^{(2)}$ are given in (13) above. Related methods for the second-order wave equation are presented by Cohen and Joly [8] and Shubin and Bell [39].

Fourier error analysis. Consider the linear convection equation, (1), on an infinite domain. A solution initiated by a harmonic function with wavenumber $\kappa$ is

$$
u(x, t)=f(t) e^{i \kappa x},
$$

where $f(t)$ satisfies the ODE

$$
\frac{d f}{d t}=-i a \kappa f
$$


If the spatial derivative is approximated by a finite-difference formula, then the ODE becomes

$$
\frac{d f}{d t}=-i a \kappa^{*} f
$$

where $\kappa^{*}$ is the modified wavenumber. For example, the modified wavenumber associated with the spatial operator given in (3) is obtained from

$$
\kappa^{*} \Delta x=\frac{a \sin (z)+(b / 2) \sin (2 z)+(c / 3) \sin (3 z)}{1+2 \alpha \cos (z)+2 \beta \cos (2 z)},
$$

where $z=\kappa \Delta x$, i.e., $z=2 \pi / \mathrm{PPW}$. The numerical phase speed, $a^{*}$, is the speed at which a harmonic function propagates numerically. It is related to the modified wavenumber by

$$
\frac{a^{*}}{a}=\frac{\kappa^{*}}{\kappa} .
$$

The dependence of $a^{*}$ on $\kappa$ introduces numerical dispersion. For the spatial operator given in (4) and (5), the modified wavenumber is obtained from

$$
\begin{aligned}
i \kappa^{*} \Delta x & =d_{0}+2\left(d_{1} \cos \kappa \Delta x+d_{2} \cos 2 \kappa \Delta x+d_{3} \cos 3 \kappa \Delta x\right) \\
& +2 i\left(a_{1} \sin \kappa \Delta x+a_{2} \sin 2 \kappa \Delta x+a_{3} \sin 3 \kappa \Delta x\right) .
\end{aligned}
$$

The real part of the modified wavenumber determines the phase (or dispersive) error, while the imaginary part determines the amplitude (or dissipative) error.

The accuracy of a time-marching method can be assessed through the characteristic polynomial of the ODE resulting from application of the time-marching method to the following ODE:

$$
\frac{d f}{d t}=\lambda f
$$

The accuracy analysis is based on the principal root of the characteristic polynomial, denoted by $\sigma(\lambda h)$, which is an approximation to $e^{\lambda h}$. In order to assess the accuracy of time-marching methods for wave propagation, we consider only pure imaginary values of $\lambda$, i.e., $\lambda=i \omega$ with $\omega$ real. The normalized local amplitude and phase errors are determined from $\sigma(\lambda h)$, as follows:

$$
\begin{aligned}
& e r_{a}=|\sigma|-1, \\
& e r_{p}=-\frac{\phi}{\omega h}+1,
\end{aligned}
$$

where $\phi=\arctan \left(\sigma_{i} / \sigma_{r}\right)$, and $\sigma_{r}$ and $\sigma_{i}$ are the real and imaginary parts of $\sigma$. Equation (16) is in the form of (21) with $\lambda=i \omega=-i a \kappa$. Substituting $\omega=-a \kappa$ into (23), we obtain

$$
e r_{p}=\frac{\phi}{z C}+1
$$

where $\sigma=\sigma(-i z C)$, and $C=a h / \Delta x$ is the Courant number. To analyze a timemarching method in combination with a spatial discretization, $\sigma=\sigma\left(-i \kappa^{*} \Delta x C\right)$, 
where $\kappa^{*}$ is the modified wavenumber associated with the spatial operator. For simultaneous space-time discretizations, the complex amplification factor is calculated directly by substituting a solution of the form $u(x, t)=\sigma^{n} e^{i \kappa x}$ into the fully discrete operator [3].

Our criterion for comparing schemes is based on the magnitude of the global amplitude and phase errors, which are

$$
\begin{aligned}
E r_{a} & =\left.|| \sigma\right|^{N}-1 \mid \\
& =\left.|| \sigma\right|^{P P W n_{w} / C}-1 \mid, \\
E r_{p} & =N|\omega h-\phi| \\
& =n_{w}\left|\frac{P P W \phi}{C}+2 \pi\right|,
\end{aligned}
$$

where $N=P P W n_{w} / C$ is the number of time steps and $n_{w}$ is the number of wavelengths traveled. Using these formulas with an accurate time-marching method and a very small Courant number gives the errors for the spatial operator alone; the time advance is effectively exact. In the results section, the various methods are compared in terms of the PPW required to keep both global phase and amplitude errors below 0.1 as a function of the number of wavelengths traveled. This error threshold is, of course, arbitrary and corresponds to that used by Lockard, Brentner, and Atkins [27]. The relative performance of the methods is similar for other choices, and thus our conclusions are independent of the specific value of the error threshold. For some schemes, especially optimized schemes, the error does not increase monotonically with the nondimensional wavenumber $z$. Hence the error can actually become larger as the grid resolution is increased. In such cases, we use the value of PPW for which all higher values of PPW also satisfy the error threshold.

Since practical problems involve multidimensional systems of equations, the use of one-dimensional scalar analysis requires some justification. A hyperbolic system of equations is diagonalizable with real eigenvalues, or wave speeds. These wave speeds can vary significantly. Consequently, the effective Courant number for the different waves can also vary significantly. A nonuniform grid further contributes to the variation in the Courant number. For the fastest wave, the Courant number must be sufficiently small such that the scheme is both accurate and stable. For the slower waves, the Courant number is then much smaller. A scheme which produces poor accuracy at low Courant numbers is thus inappropriate for such problems. This situation can arise if a scheme relies on cancellation of time and space errors to achieve high accuracy. For example, several schemes which combine the spatial and temporal discretization produce the perfect-shift property at specific Courant numbers. Often this perfect cancellation of temporal and spatial errors is achieved at a Courant number of unity. For such schemes, the error increases as the Courant number is reduced since the temporal error decreases and no longer cancels the spatial error. Hence it is important to assess schemes over a range of Courant numbers. Note that this is not an issue with schemes which combine a high-accuracy spatial discretization with a high-accuracy time-marching method. Since such schemes generally do not rely on cancellation to achieve high accuracy, the error does not increase as the Courant number is reduced.

The situation is similar in multidimensions. For most spatial discretizations, the error is largest for waves propagating at 0 or 90 degrees to the grid [46, 23, 53]. The 


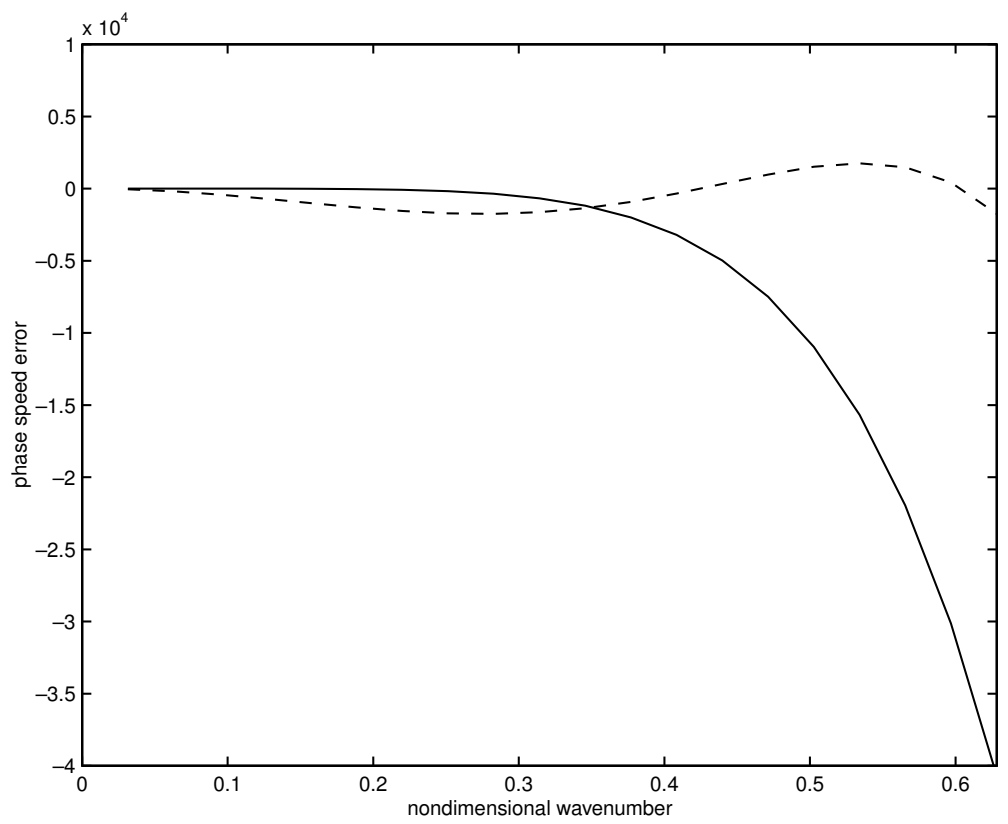

FIG. 1. Phase speed error for the maximum-order (-) and optimized (- - ) schemes of Zingg, Lomax, and Jurgens [55].

error from the time-marching method is isotropic. Therefore, if there is no cancellation of spatial and temporal errors, the one-dimensional analysis is conservative. In contrast, if a scheme relies on such cancellation, then the error at arbitrary angles of propagation can be much higher than that in one dimension. For example, a scheme with the perfect shift property at a Courant number of unity in one dimension can produce large errors at the same Courant number in multidimensions and can even be unstable. This occurs because the spatial error is anisotropic while the temporal error is isotropic. Hence they cannot cancel at all angles. Fortunately, this situation can be revealed by a one-dimensional analysis as long as a wide range of Courant numbers is considered. As the Courant number tends to zero, so does the temporal error, and only the spatial error remains. Hence the one-dimensional analysis at a low Courant number can represent the worst case for some schemes, since there is no cancellation of temporal and spatial errors. This is confirmed by the results presented below.

Before proceeding to the results, we briefly discuss the concept of an optimized scheme, using the schemes of Zingg, Lomax, and Jurgens [55] as an example. Figure 1 shows the phase speed error for the maximum-order and optimized spatial discretizations given in [55] for a nondimensional wavenumber $z$ between 0 and $\pi / 5$, i.e., for waves resolved with at least $10 \mathrm{PPW}$. This optimized scheme trades increased errors at low wavenumbers in return for greatly reduced errors for $0.4 \leq z \leq \pi / 5$. The idea is to extend the range of wavenumbers for which the scheme is sufficiently accurate. Note that, for the optimized scheme, the error at $z=0.29$ (22 PPW) is very close to that at $z=\pi / 5(10 \mathrm{PPW})$. Consequently, there is no benefit in increasing the grid resolution from $10 \mathrm{PPW}$ to $22 \mathrm{PPW}$. If a grid resolution of $10 \mathrm{PPW}$ is insufficient for a given propagation distance, then greater than $22 \mathrm{PPW}$ will be required. This explains the sudden jumps in the grid resolution requirements of optimized schemes which will be seen below. 


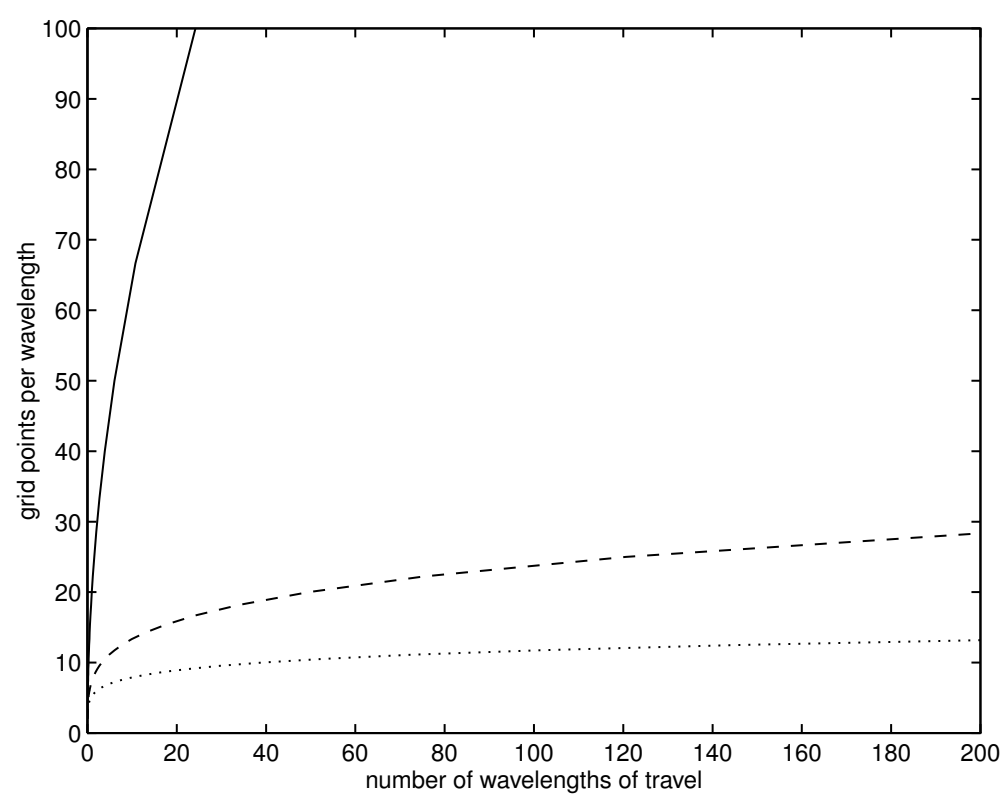

FIG. 2. Grid resolution requirements for second-order (-), fourth-order (- - -), and sixth-order (..) noncompact centered spatial differences.

\section{Results.}

Spatial difference operators. In this section, we consider the errors produced by the spatial operators alone. The figures show the PPW required to keep both global phase and amplitude errors below 0.1 as a function of the number of wavelengths traveled. Figure 2 shows the grid resolution requirements for second-, fourth-, and sixth-order noncompact centered difference schemes. Since these spatial operators are nondissipative, the grid resolution requirements are determined from the global phase error. The requirements of the second-order scheme are clearly excessive. While more accurate second-order discretizations are available, such as the FDTD scheme or the upwind leapfrog scheme [33], none of these meets our criterion of 30 PPW for 200 wavelengths of travel except under specific conditions.

The PPW requirements for compact centered difference schemes of up to tenth order are shown in Figure 3. The tenth-order scheme requires the solution of a pentadiagonal system of equations. The remaining schemes lead to tridiagonal systems. The compact schemes are considerably more accurate than the noncompact schemes of equivalent order.

We next demonstrate the tradeoffs associated with optimization, using the tridiagonal five-point operators $(\beta=c=0)$ of Haras and Ta'asan as an example. Figure 4 shows the behavior of three different optimized schemes given in [15] as well as the maximum-order scheme which can be obtained using this operator, which is sixthorder. Note the presence of sudden jumps in the grid resolution requirements of the optimized schemes. These are associated with the fact that for an optimized scheme the error does not increase monotonically with increasing wavenumber, as discussed earlier. Their location is dependent on the error threshold used. Reducing the error allowed moves the jumps to the left. With the present error threshold, the scheme denoted "A" is superior up to a distance of travel of about 60 wavelengths. Scheme B 


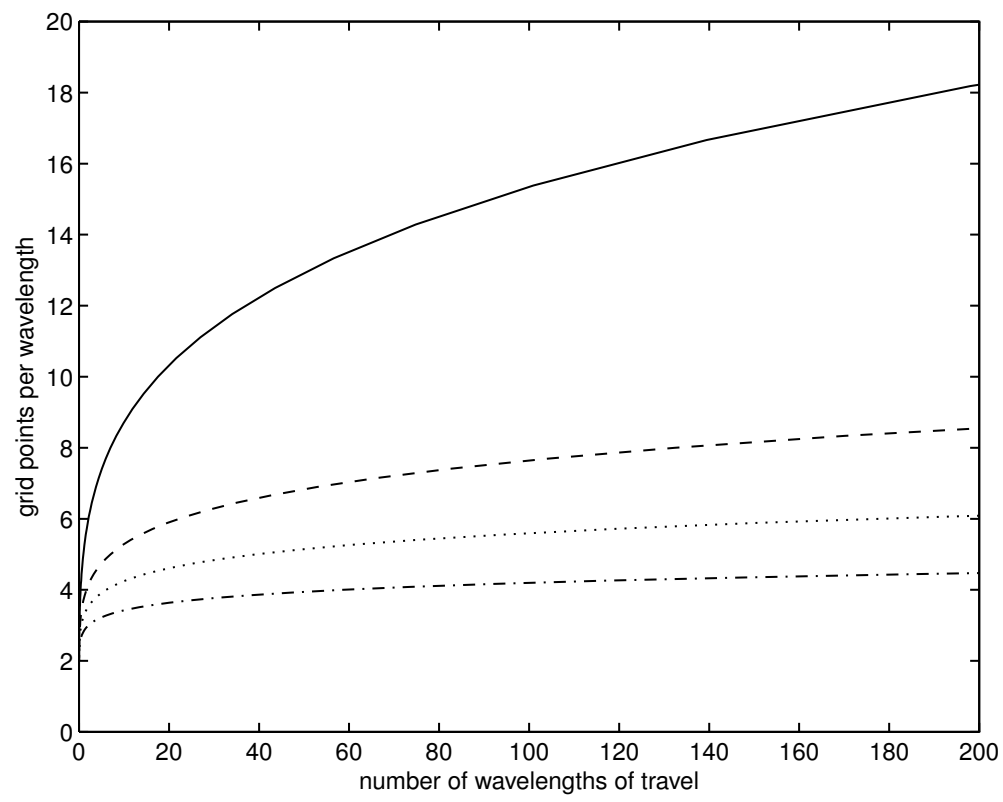

FIG. 3. Grid resolution requirements for fourth-order (-), sixth-order (- - -), eighth-order $(\cdots)$, and tenth-order $(-\cdot-)$ compact centered spatial differences.

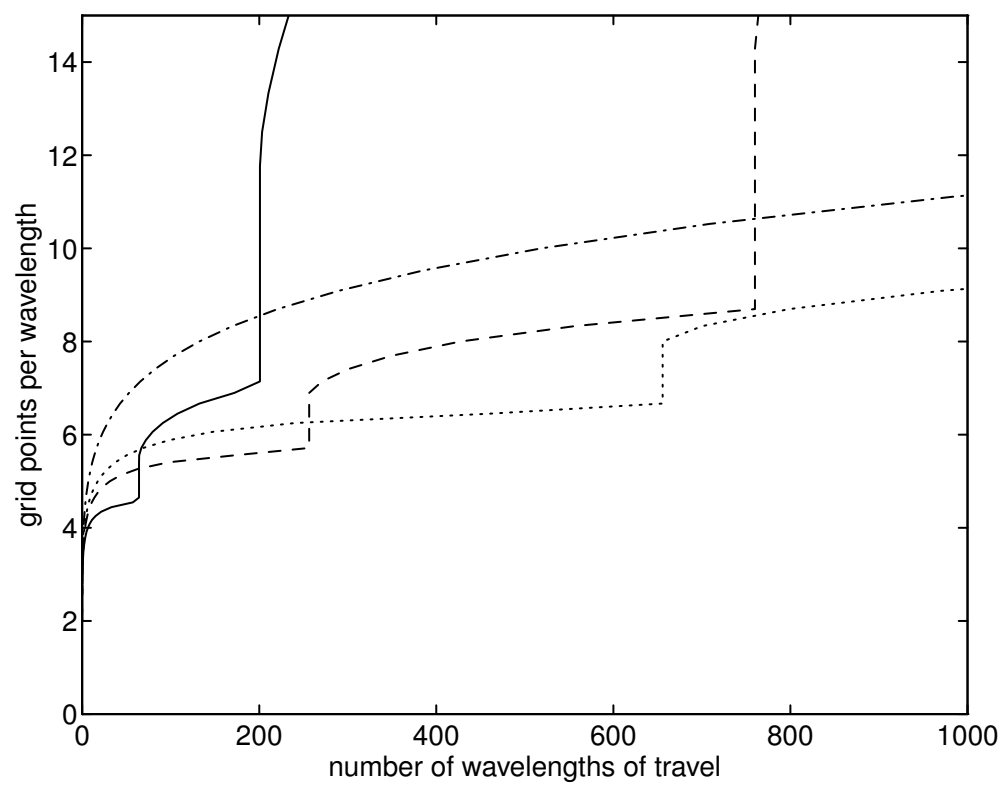

FIG. 4. Grid resolution requirements for the tridiagonal spatial operators with 5-point righthand stencil of Haras and Ta'asan [15]: scheme A (-), scheme B (- - ), scheme C ( ..), sixth-order scheme $(-\cdot-)$.

is superior for distances up to 250 wavelengths while scheme $\mathrm{C}$ is preferable for even longer distances. Such behavior is typical of optimized schemes. Aggressive optimization (as in the case of scheme A) leads to excellent performance for small distances 
of travel but poor performance for longer distances. In this paper we concentrate on distances of travel up to 200 wavelengths. Hence we consider only scheme B further.

Figure 5 shows the PPW required by the optimized schemes of Haras and Ta'asan for distances of travel up to 200 wavelengths. In each case, the best scheme presented by Haras and Ta'asan for this distance of travel is shown, as discussed above. The pentadiagonal scheme requires about $3.7 \mathrm{PPW}$ for 200 wavelengths of travel while the tridiagonal seven-point scheme requires 4.5 and the tridiagonal five-point scheme requires 5.7. The computational effort is roughly proportional to $P P W^{d+1}$, where $d$ is the number of dimensions, since the number of grid nodes is proportional to $P P W^{d}$, and the additional factor of PPW is associated with the decrease in the time step required to maintain a constant Courant number as PPW is increased, i.e., $N=P P W n_{w} / C$. Therefore the increased cost per grid node of the pentadiagonal scheme is not justified. However, the tridiagonal seven-point scheme is more efficient than the tridiagonal five-point scheme.

The noncompact optimized schemes of Lockard, Brentner, and Atkins [27], Zingg, Lomax, and Jurgens [55], and Tam and Webb [43] are compared with sixth-order centered differences in Figure 6. For the scheme of Lockard, Brentner, and Atkins [27], the PPW requirements are determined by the amplitude error. For the scheme of Zingg, Lomax, and Jurgens [55], the phase and amplitude errors produce roughly equivalent grid resolution requirements over this distance. Since Tam and Webb's scheme [43] is nondissipative, its PPW requirements are determined by the phase error. The figure shows that Tam and Webb's scheme [43] has been optimized for fairly small distances of travel, leading to excellent performance for less than roughly 14 wavelengths of travel. The scheme of Lockard, Brentner, and Atkins [27] requires roughly 8 PPW for 200 wavelengths of travel while that of Zingg, Lomax, and Jurgens [55] requires over 9 PPW.

Figure 7 shows the PPW requirements of the staggered spatial operators. In each case, the staggered schemes are much more accurate than their nonstaggered counterparts of equivalent order. The grid requirements of the second-order scheme are again excessive. However, when used with staggered leapfrog time marching (the FDTD scheme), better results can be obtained. Also shown in Figure 7 is an optimized scheme with $b_{3}=103 / 19200, b_{2}=-1315 / 19200$, and $b_{1}=22630 / 19200$, which produces excellent performance for up to 200 wavelengths of travel.

Time-marching methods. Figures 8 and 9 show the amplitude and phase errors produced by the four time-marching methods under consideration. The five- and six-stage methods shown are the maximum-order versions rather than the optimized methods, which are discussed in the next subsection. In order to account for the number of stages in the Runge-Kutta methods, the errors are plotted versus $\omega h / p$, where $p$ is the number of stages. Hence the errors shown are for approximately equal computational effort. Since the time step of a $p$-stage scheme is thus $p$ times larger than that of a single-stage scheme, the amplitude error shown is $|\sigma|^{1 / p}-1$.

The figures show that the phase errors produced by these methods are larger than the amplitude errors. Each increase in the order of the Runge-Kutta method produces an increase in accuracy even though the extra work has been accounted for. The fourth-order Adams-Bashforth method is much more accurate than the fourthorder Runge-Kutta method per unit cost. It produces the lowest amplitude error of the methods considered and phase error comparable to the five-stage Runge-Kutta method.

In order to compare time-marching methods properly, one must consider the 


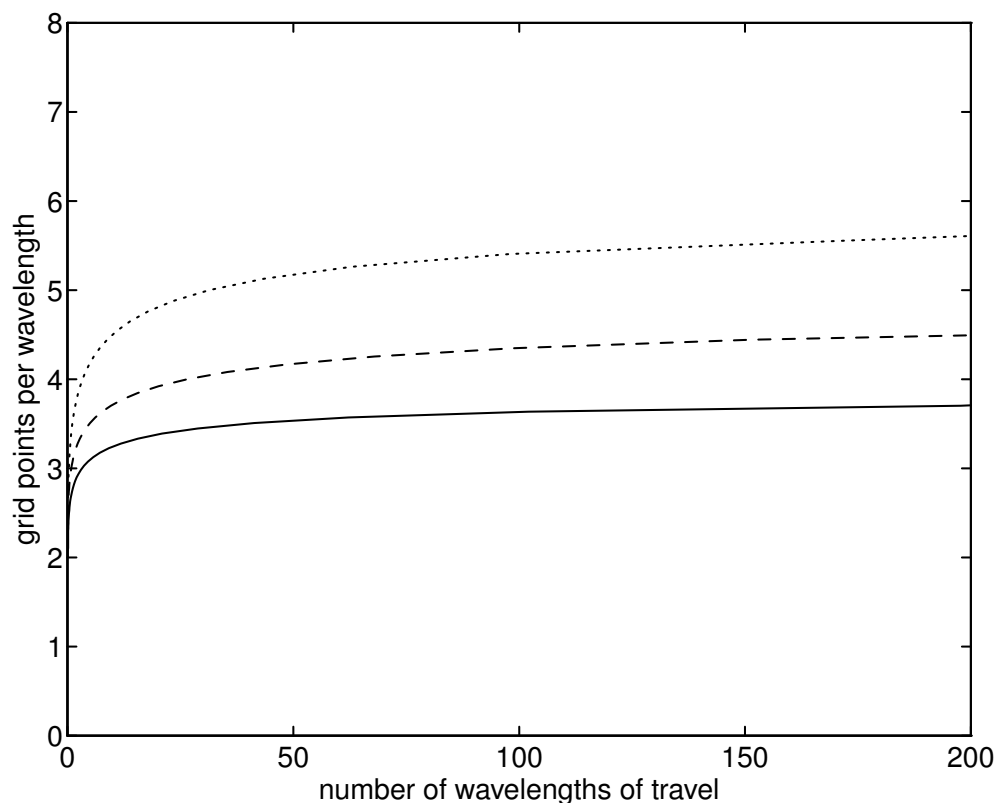

FIG. 5. Grid resolution requirements for the spatial operators of Haras and Ta'asan [15]: pentadiagonal operator with seven-point right-hand stencil (-), tridiagonal operator with seven-point right-hand stencil (- - -), tridiagonal operator with five-point right-hand stencil (...).

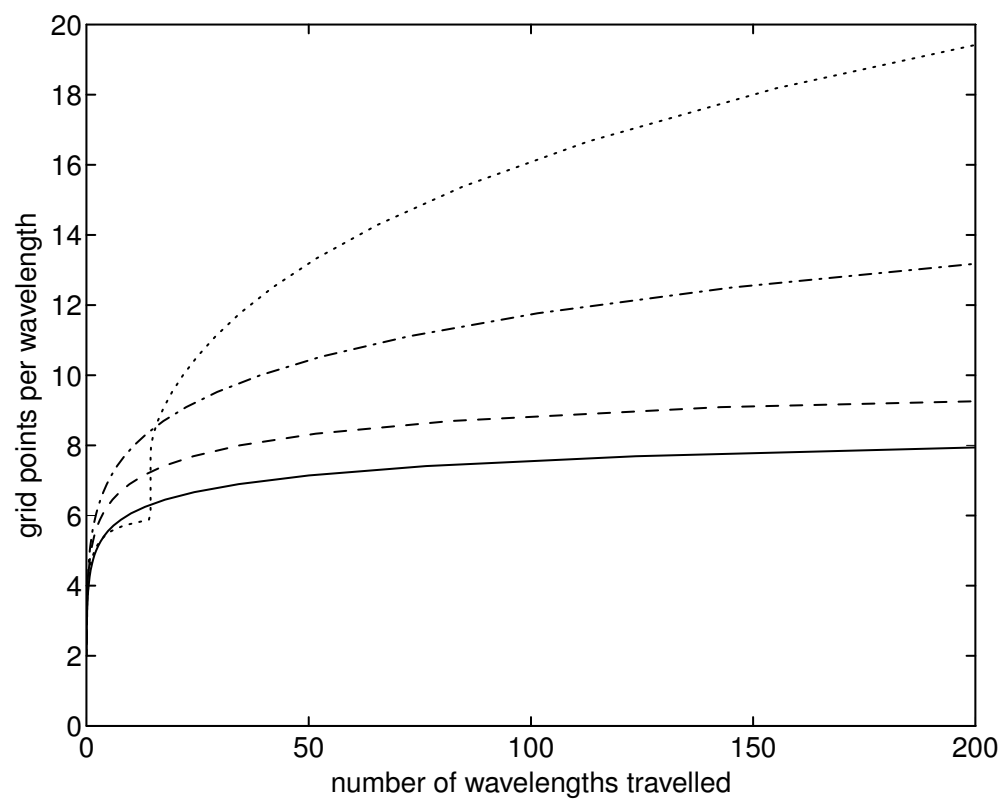

FIG. 6. Grid resolution requirements for the spatial operators of Lockard, Brentner, and Atkins [27] (-), Zingg, Lomax, and Jurgens [55] (- - ), Tam and Webb [43] (. .), and sixthorder centered differences $(-\cdot-)$.

spatial discretization to be used. For each combination of a spatial discretization and a time-marching method, there is a Courant number which minimizes the computational 


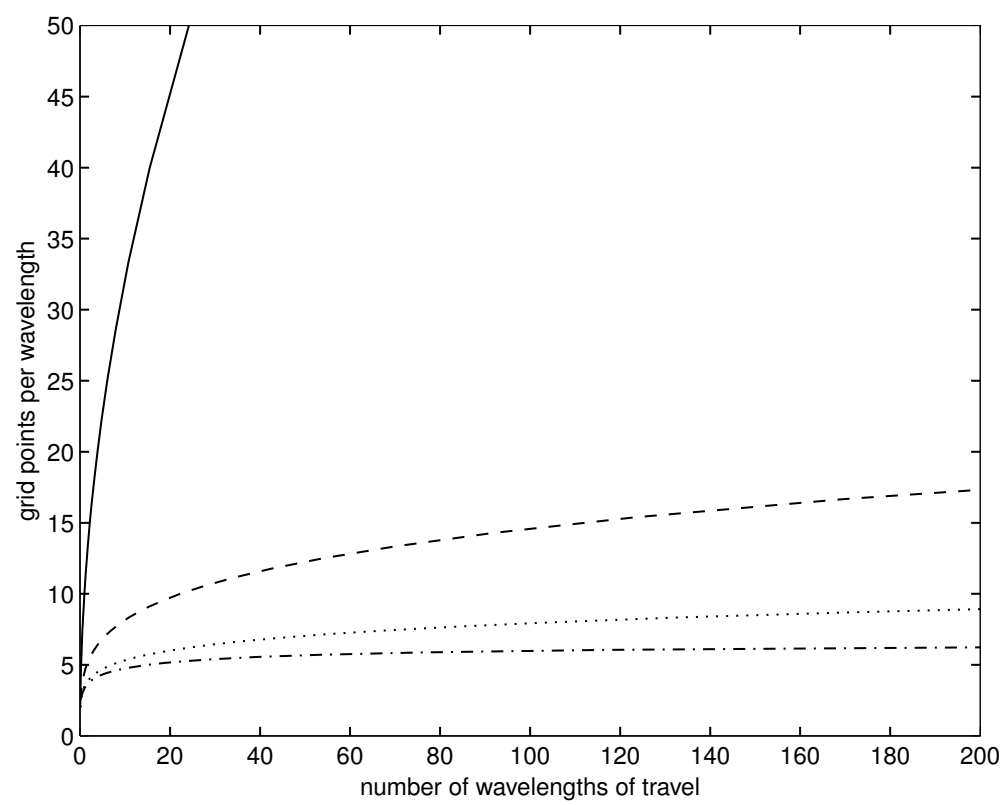

FIG. 7. Grid resolution requirements for second-order (-), fourth-order (- - -), sixth-order $(\cdots)$, and optimized $(-\cdot-)$ centered spatial differences on a staggered grid.

work to achieve a given standard of accuracy for a specified distance of travel. The computational work is proportional to the product of the number of derivative function evaluations and the work per function evaluation. The former is given by $p N=$ $p P P W n_{w} / C$, while the latter is proportional to the number of grid nodes, $P P W^{d}$, where $p$ is again the number of stages (which gives the number of function evaluations per time step for the schemes under consideration) and $d$ the number of dimensions. Hence the computational work for a given distance of travel, $n_{w}$, is proportional to

$$
F=\frac{p}{C} P P W^{d+1} .
$$

For example, with fourth-order centered differences in space the optimum Courant number for the fourth-order Runge-Kutta method in two dimensions (determined by trial and error) is about 1.25 for 200 wavelengths of travel and global errors less than 0.1. With this spatial operator, the optimum Courant number for the AdamsBashforth method is determined by its stability bound, which for pure imaginary $\lambda$ is roughly 0.43 . With fourth-order centered differences in two dimensions, the resulting Courant number is about 0.21 . The value of $F$ produced at this Courant number is slightly higher than that produced using the fourth-order Runge-Kutta method at its optimum Courant number. Since the fourth-order Runge-Kutta method also requires less memory than the fourth-order Adams-Bashforth method, it is clearly the preferred method for use with fourth-order centered differences in space.

With sixth-order centered differences in space, the comparison changes. The optimum Courant number for the fourth-order Runge-Kutta method is reduced to roughly $2 / 3$ while that for the fourth-order Adams-Bashforth method remains stability limited, leading to a Courant number of roughly 0.19. In this case, the value of $F$ produced by the Adams-Bashforth method is less than $80 \%$ of that for the Runge-Kutta method. Thus the comparison is more complicated, as one must weigh 


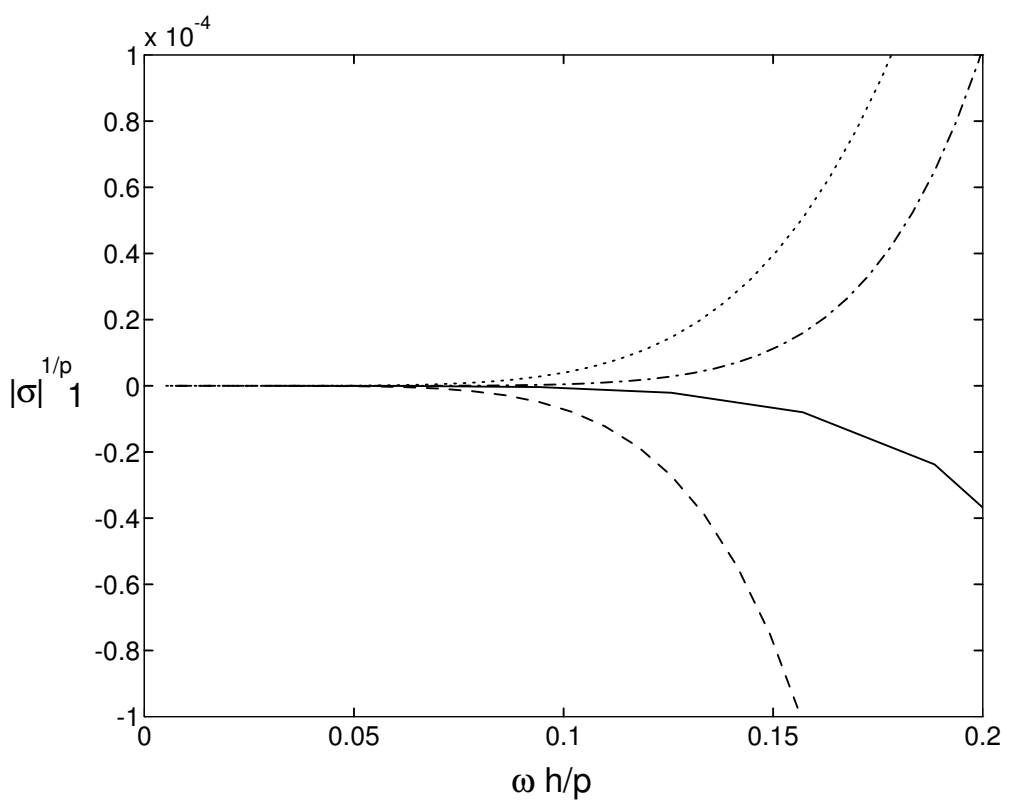

FIG. 8. Amplitude error produced by the fourth-order Adams-Bashforth method (-), the fourth-order Runge-Kutta method (- - ), the five-stage Runge-Kutta method (...), and the sixstage Runge-Kutta method (-.-).

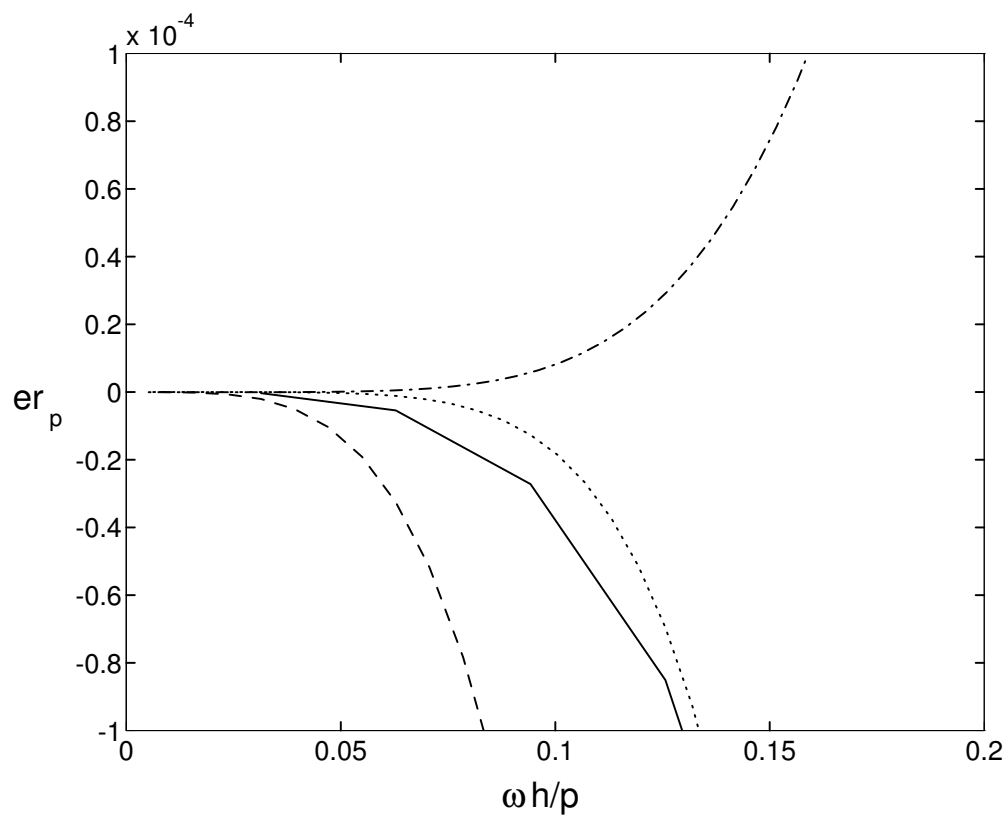

Fig. 9. Phase error produced by the fourth-order Adams-Bashforth method (-), the fourthorder Runge-Kutta method (- - -), the five-stage Runge-Kutta method (...), and the six-stage Runge-Kutta method (- - - ).

the increased efficiency of the Adams-Bashforth method against the reduced memory requirements of the Runge-Kutta method.

The difficulty with the maximum-order five- and six-stage Runge-Kutta methods 


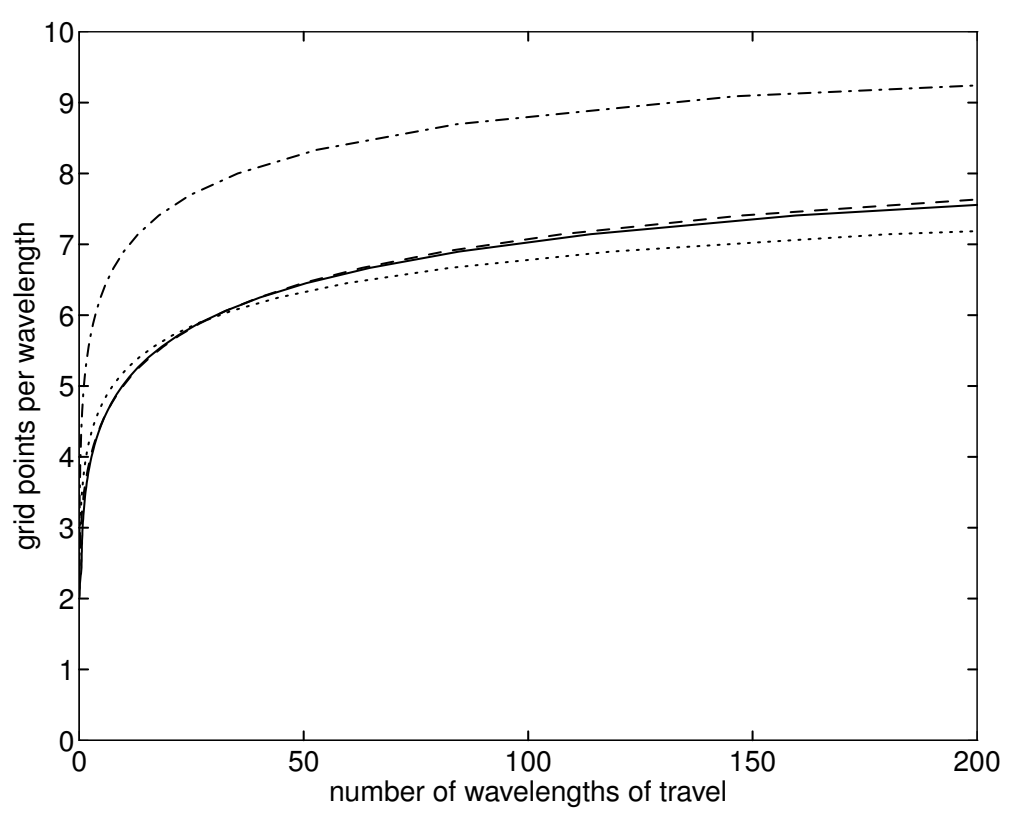

FIG. 10. Grid resolution requirements for the spatial and temporal operators of Haras and Ta'asan [15] at a Courant number of 0.9: pentadiagonal operator with seven-point right-hand stencil (-), tridiagonal operator with seven-point right-hand stencil (- - -), tridiagonal operator with fivepoint right-hand stencil (...); spatial and temporal operators of Zingg, Lomax, and Jurgens [55] at a Courant number of $1(-\cdot-)$.

is that they are unstable for pure imaginary $\lambda$, as shown in Figure 8. We consider these schemes further below.

Combined space-time discretizations. Haras and Ta'asan modified the coefficients of the five-stage Runge-Kutta method to produce stability for pure imaginary $\lambda$ while maintaining second-order accuracy and optimized error behavior. The methods are designed for $C=0.9$. Figure 10 shows the grid resolution requirements of the three spatial operators of Haras and Ta'asan compared in Figure 5 in combination with their five-stage time-marching method at a Courant number of 0.9. All three schemes require between 7 and $8 \mathrm{PPW}$ for 200 wavelengths of travel. The advantage of the more accurate spatial operators has been lost. Either a lower Courant number or a more accurate time-marching method should be used.

As a result of the dissipation in the spatial operator, the six-stage time-marching method of Zingg, Lomax, and Jurgens [55] is stable up to a Courant number a little greater than unity in two dimensions. The grid requirements for the combined spacetime discretization at a Courant number of unity are shown in Figure 10. Comparison with Figure 6 shows that the time-marching method introduces very little error compared to the spatial differencing. Tam and Webb [43] use an optimized four-step Adams-Bashforth method in conjunction with their spatial operator. It produces little error for Courant numbers less than about 0.3. In both cases, optimization of the time-marching method has a much smaller impact than optimization of the spatial operator.

Figure 11 shows the PPW requirements of the fourth-order staggered spatial difference operator combined with staggered leapfrog time marching. As the Courant number is increased from 0.001 to 0.1 , the PPW requirements decrease, since the 


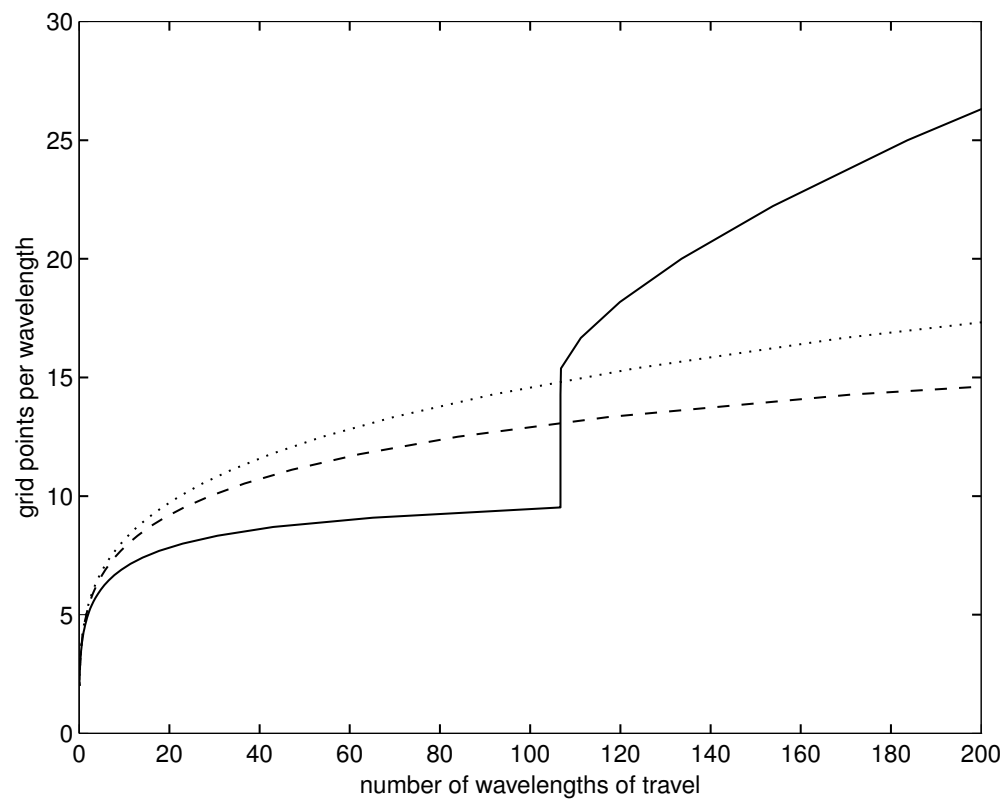

FIG. 11. Grid resolution requirements for fourth-order centered differences on a staggered grid coupled with staggered leapfrog time marching at Courant numbers of 0.2 (-), 0.1 (- - -), and 0.001 $(\cdots)$.

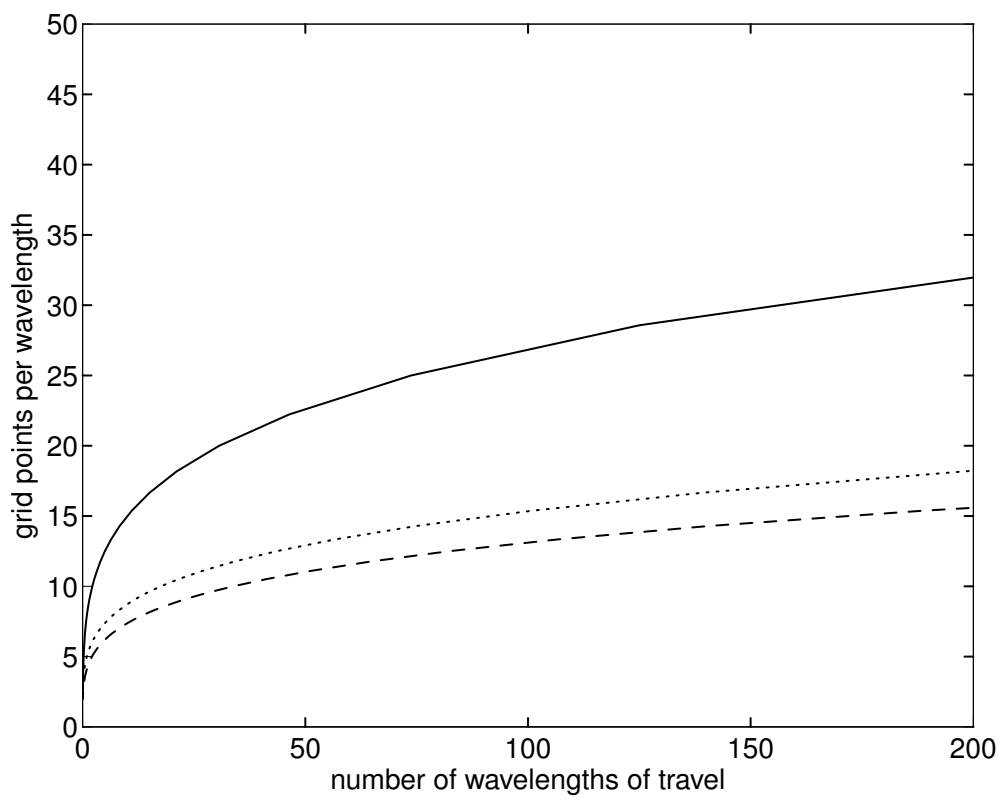

FIG. 12. Grid resolution requirements for the scheme of Davis [10] at Courant numbers of 3 (-), $1.5(--)$, and $0.01(\cdots)$.

error from the time-marching method is of opposite sign to that of the spatial operator. However, as the Courant number is further increased, the error from the second-order time-marching method begins to dominate. Excellent performance for 
200 wavelengths of travel is obtained for Courant numbers up to 0.1.

Results for the method of Davis [10] are shown in Figure 12. Since the method is nondissipative, the PPW requirements are determined from the phase error. This scheme is unconditionally stable. For Courant numbers under 1.5, less than $19 \mathrm{PPW}$ are required for 200 wavelengths of travel. While this is quite good, much better than many schemes, it is not sufficient to justify the additional computational effort associated with an implicit scheme.

Figure 13 shows the grid resolution requirements for the Gottlieb-Turkel scheme, which is stable up to a Courant number of $2 / 3$ in one dimension. This scheme is very robust and has received extensive use in nonlinear applications. Since the method is only second-order in time, high accuracy is obtained only for Courant numbers less than about 0.13 . Less than $29 \mathrm{PPW}$ are required to propagate a wave 200 wavelengths with Courant numbers below this value. Although the cost per grid node is reasonably low, these PPW requirements are too high for efficient simulation over such distances.

Finally, the grid requirements of the nondissipative two-step explicit scheme, (14), are shown in Figure 14. This scheme is stable up to a Courant number of unity in one dimension. It has the perfect shift property at this Courant number. The phase error increases as the Courant number is reduced. Less than $29 \mathrm{PPW}$ are required for all stable Courant numbers. Since it provides reasonably low error at Courant numbers up to unity, this scheme has a very low cost per grid node, substantially lower than the Gottlieb-Turkel scheme. However, the PPW requirements are again much higher than some of the other schemes considered.

A numerical example. In this section, we present a numerical simulation of the propagation and reflection of an electromagnetic wave which demonstrates the applicability of the previous analysis in multidimensional cases using nonuniform curvilinear grids. Further details, including the treatment of the boundary conditions, are available in [19]. The governing equations are the transverse magnetic set of the two-dimensional time-domain Maxwell equations. The simulation consists of a pulsed plane wave incident on a perfectly-conducting cylinder. A grid containing 5,400 nodes is shown in Figure 15. Figure 16 shows a snapshot of the electric field intensity computed on a grid with 21,600 nodes using the maximum-order version of the method of Zingg, Lomax, and Jurgens $[52,55]$. The dashed contours indicate negative values of the electric field intensity. This solution is visually indistinguishable from that computed using the same method on a grid with 16 times as many nodes, which is used as a baseline to estimate numerical errors. The reflected wave has not yet reached the outer boundary, so spurious reflections are not an issue.

Figures 17 and 18 show the $L_{2}$ norm of the numerical error versus the number of grid nodes and the CPU effort, respectively. Four methods are included, the maximum-order (MO) and optimized (O10) schemes of Zingg, Lomax, and Jurgens $[52,55]$, as well as second- (C2) and fourth-order (C4) centered differences. The second- and fourth-order difference schemes are combined with fourth-order RungeKutta time marching and include a very small amount of artificial dissipation for stability. These figures show that the higher-order and optimized methods lead to substantial reductions in both memory and CPU time. The relative grid resolution requirements of the methods are consistent with the predictions obtained from Fourier analysis. Furthermore, the results show that the increased cost per grid node of the higher-order methods is insignificant compared to the reduction in grid resolution required. The benefits of the optimized scheme over the maximum-order scheme are fairly modest due to the nature of the waveform, which is a Gaussian and thus has 


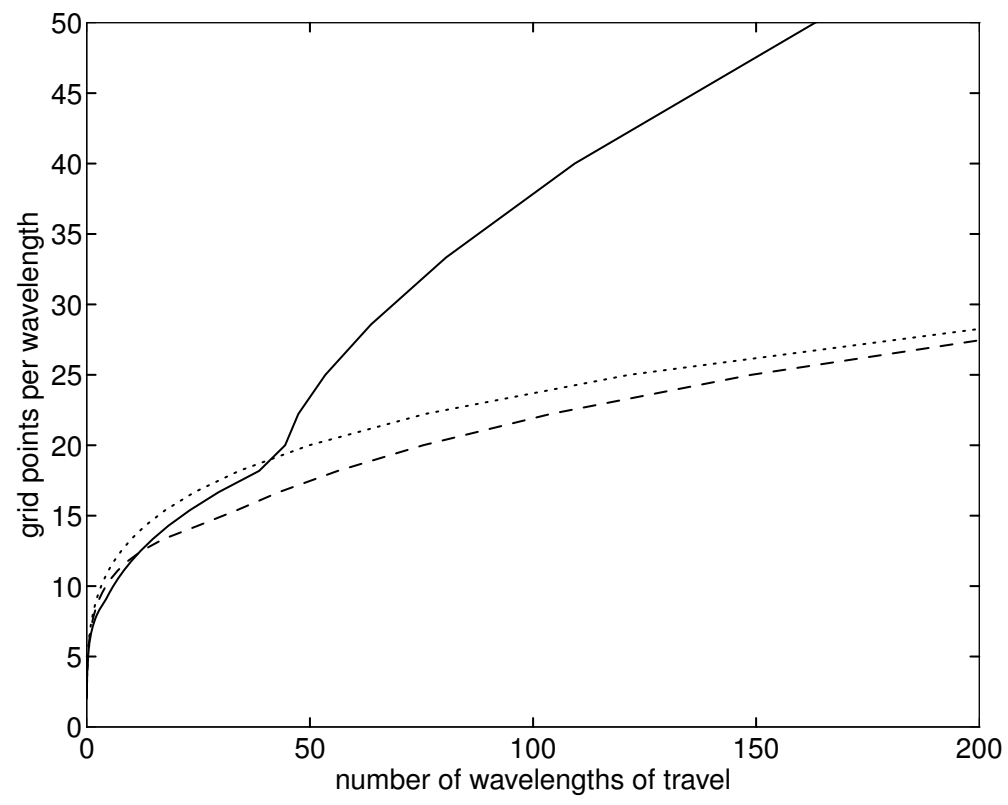

FIG. 13. Grid resolution requirements for the scheme of Gottlieb and Turkel [13] at Courant numbers of 0.2 (-), $0.13(--)$, and $0.01(\cdots)$.

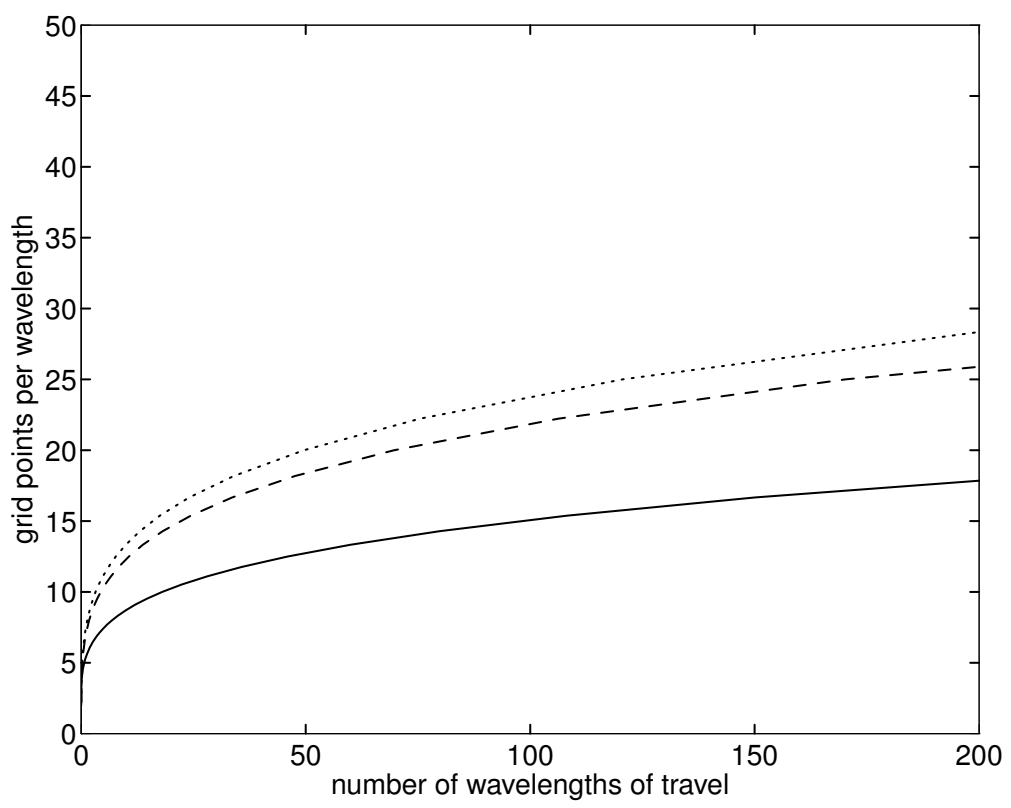

FIG. 14. Grid resolution requirements for the two-step explicit scheme (10) at Courant numbers of 0.9 (-), $0.5(--)$, and $0.01(\cdots)$.

considerable low-wavenumber content.

Discussion and conclusions. Fourier analysis shows that optimized schemes can provide a significant advantage over their maximum-order counterparts. How- 


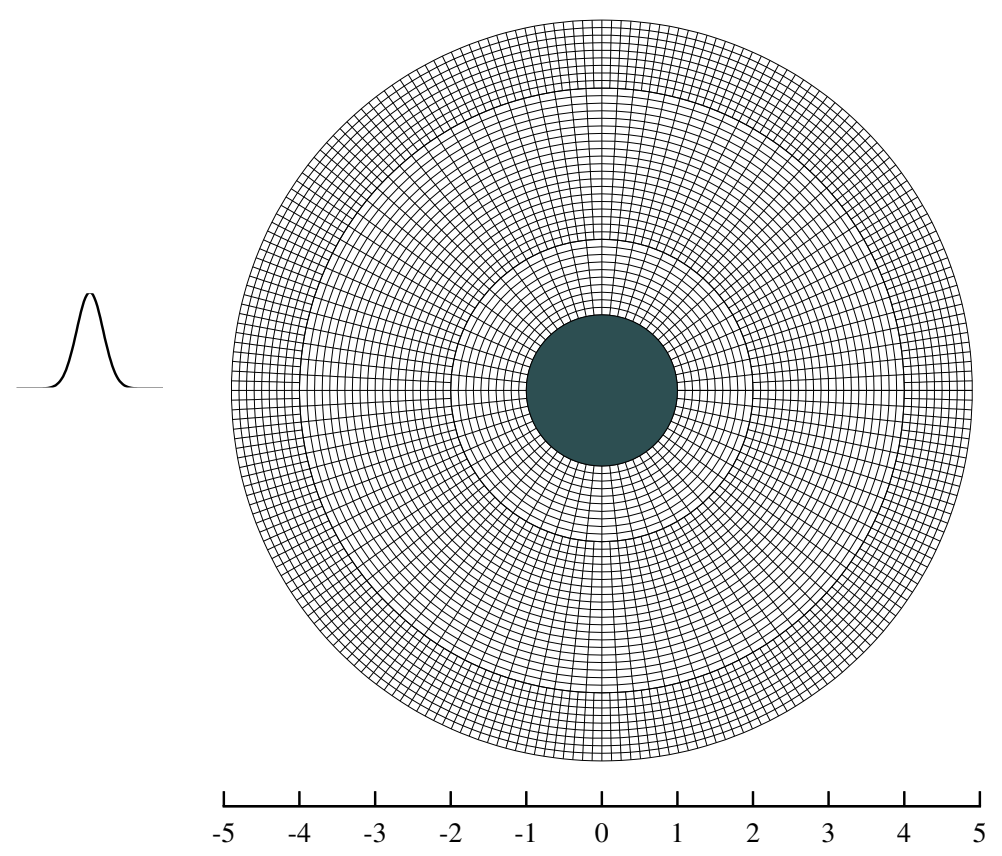

FIG. 15. Grid for the perfectly-conducting cylinder.

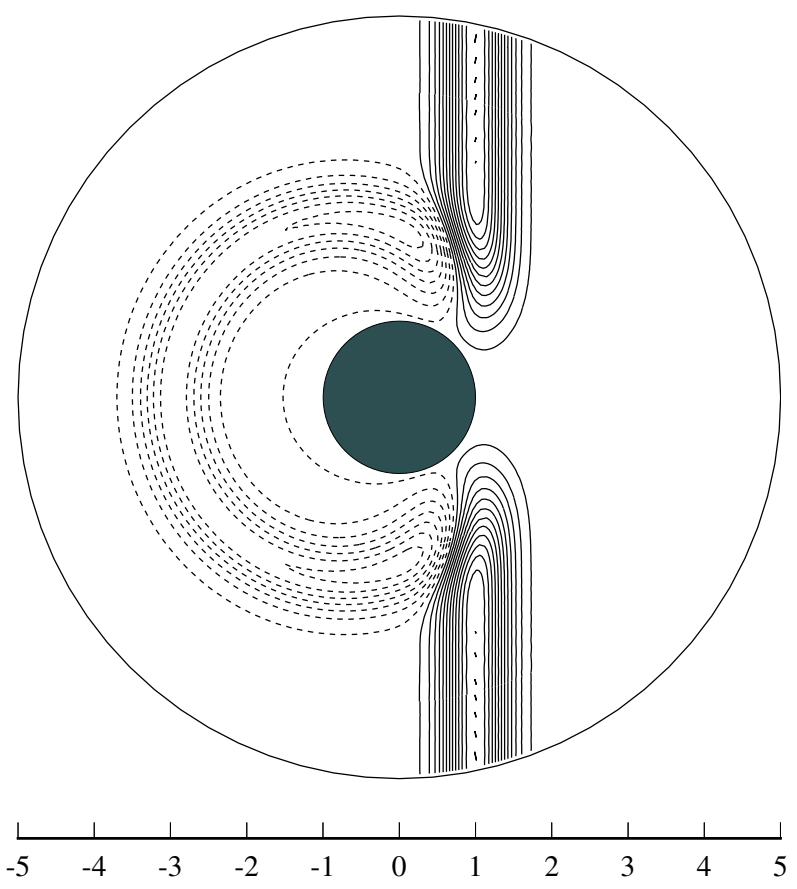

FIG. 16. Computed contours of electric field intensity. 


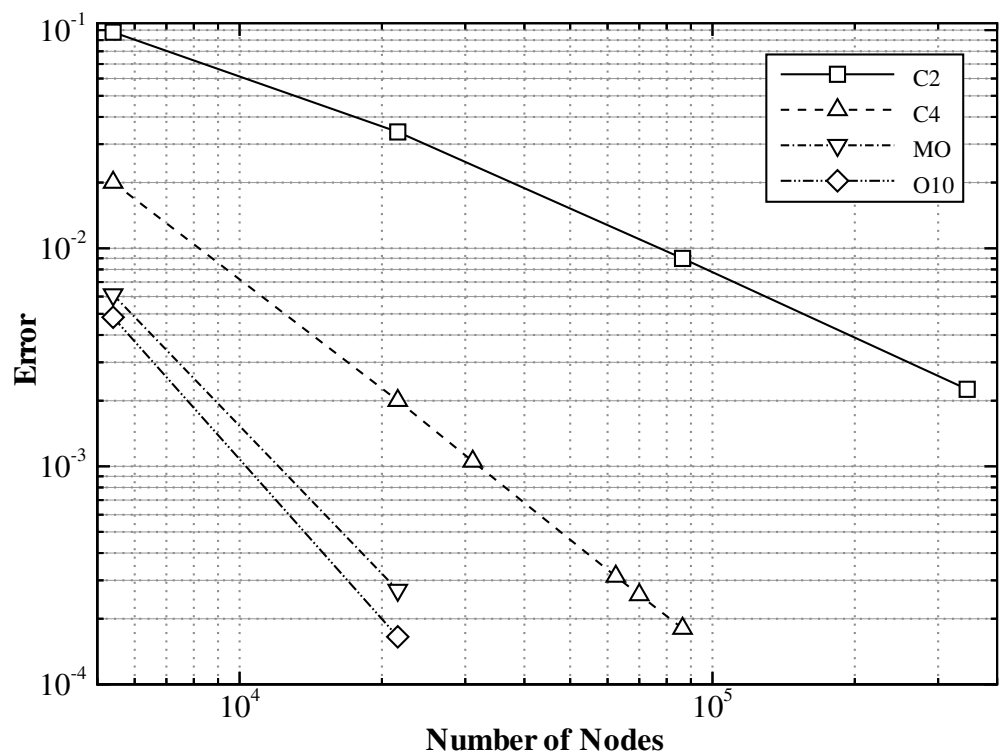

FIG. 17. Error in the electric field intensity as a function of the number of grid nodes.

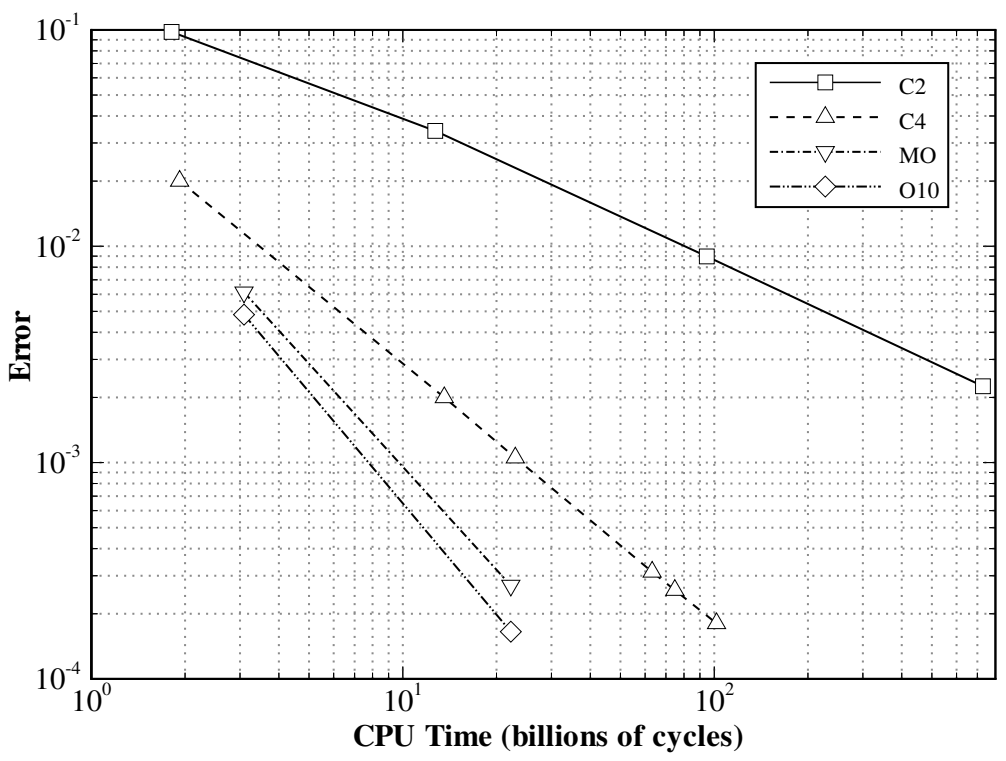

FIG. 18. Error in the electric field intensity as a function of the CPU time.

ever, if optimized too aggressively, they perform poorly for longer distances of travel. Furthermore, if a waveform has significant low wavenumber content, as in the case of a Gaussian pulse, the benefits of an optimized scheme can be minimal, and maximumorder schemes can even be superior. The spatial operators of Haras and Ta'asan [15], Lockard, Brentner, and Atkins [27], and Zingg, Lomax, and Jurgens [52, 55] all provide adequate accuracy for 200 wavelengths of travel. The selection of a time-marching method is not quite as critical, since the computational work varies linearly with the time-step size. Consequently, the cost of reducing the time step is much less than the 
cost of reducing the PPW, and, furthermore, has no memory implications. AdamsBashforth and low-storage Runge-Kutta methods can be used, with the latter often preferable because of their reduced memory requirements.

For appropriate problems, such as those involving electromagnetic waves or acoustic waves in a quiescent medium, staggered spatial schemes perform very well. These can be combined with either a high-order time-marching method or the staggered leapfrog method. In the latter case, a low Courant number should be used. For large propagation distances, higher-order time-marching methods are more efficient.

The grid requirements of the explicit fourth-order methods involving simultaneous space-time discretization are reasonably low considering the low cost of these schemes, especially the two-step explicit scheme. This suggests that sixth-order and optimized extensions of these schemes are worthy of investigation.

Based on the results presented, it is clear that high-order and optimized finitedifference methods will play an important role in the simulation of high-frequency linear wave phenomena. Several of the methods studied have the potential to substantially reduce the computational requirements for accurate simulations, including both CPU time and memory. The choice of an optimization strategy can be correlated with a specific distance of propagation. Among the various optimized schemes of Haras and Ta'asan, for example, specific choices can be made if the propagation distance can be estimated. This suggests the use of optimized schemes which are specifically tailored to the spectral content and distance of propagation of a given simulation. In addition to providing a useful reference for scheme evaluation and comparison, our results can be used as a basis for selecting an appropriate grid resolution when applying these schemes.

Appendix. The following are the coefficients of the finite-difference schemes studied. The number of significant figures given is based on the number given in the cited references.

The spatial operator of Haras and Ta'asan is given in (3). The schemes shown in Figure 4 have $\beta=c=0$. The remaining coefficients are

Scheme A:

$$
\begin{aligned}
\alpha & =0.3534620453 \\
a & =1.566965775 \\
b & =0.1399583152
\end{aligned}
$$

Scheme B:

$$
\begin{aligned}
\alpha & =0.3461890571, \\
a & =1.5633098070, \\
b & =0.1290683071 ;
\end{aligned}
$$

Scheme C:

$$
\begin{aligned}
\alpha & =0.3427812069, \\
a & =1.5614141543, \\
b & =0.124148259 .
\end{aligned}
$$

In Figure 5, the pentadiagonal seven-point scheme has

$$
\alpha=0.5801818925,
$$




$$
\begin{aligned}
\beta & =0.0877284887, \\
a & =1.3058941939, \\
b & =0.9975884963, \\
c & =0.0323380724 .
\end{aligned}
$$

The tridiagonal seven-point scheme has

$$
\begin{aligned}
\alpha & =0.3904091387, \\
\beta & =0, \\
a & =1.5638887738, \\
b & =0.2348222711, \\
c & =-0.0178927675 .
\end{aligned}
$$

The tridiagonal five-point operator is scheme B above.

In Figure 6, the scheme of Lockard, Brentner, and Atkins is the average of two schemes with the following coefficients, as defined in (6):

$$
\begin{aligned}
a_{-4} & =0.0207860419, \\
a_{-3} & =-0.1500704734, \\
a_{-2} & =0.5234309723, \\
a_{-1} & =-1.34207332539, \\
a_{0} & =0.574548248808, \\
a_{1} & =0.4090357053658, \\
a_{2} & =-0.035657169508
\end{aligned}
$$

and

$$
\begin{aligned}
& a_{0}=0 \\
& a_{1}=-a_{-1}=0.763289242273 \\
& a_{2}=-a_{-2}=-0.160631393818 \\
& a_{3}=-a_{-3}=0.019324515121 .
\end{aligned}
$$

The optimized scheme of Zingg, Lomax, and Jurgens [52, 55] is given by (4) and (5) with the following coefficients:

$$
\begin{aligned}
& a_{1}=0.75996126 \\
& a_{2}=-0.15812197 \\
& a_{3}=0.018760895 \\
& d_{0}=0.1 \\
& d_{1}=-0.076384622, \\
& d_{2}=0.032289620 \\
& d_{3}=-0.0059049989 .
\end{aligned}
$$

Tam and Webb's scheme is obtained from (4) with (coefficients are from [42])

$$
\begin{aligned}
& a_{1}=0.770882380518, \\
& a_{2}=-0.166705904415, \\
& a_{3}=0.0208431427703 .
\end{aligned}
$$


The five-stage Runge-Kutta method shown in Figures 8 and 9 has the following characteristic polynomial:

$$
\sigma(\lambda h)=1+\lambda h+\frac{(\lambda h)^{2}}{2}+\frac{(\lambda h)^{3}}{6}+\frac{(\lambda h)^{4}}{24}+\frac{(\lambda h)^{5}}{120} .
$$

The six-stage method is obtained from (8) with $\alpha_{5}=1 / 2, \alpha_{4}=1 / 3, \alpha_{3}=1 / 4$, $\alpha_{2}=1 / 5, \alpha_{1}=1 / 6$, leading to the following characteristic polynomial:

$$
\sigma(\lambda h)=1+\lambda h+\frac{(\lambda h)^{2}}{2}+\frac{(\lambda h)^{3}}{6}+\frac{(\lambda h)^{4}}{24}+\frac{(\lambda h)^{5}}{120}+\frac{(\lambda h)^{6}}{720} .
$$

The characteristic polynomial of the optimized five-stage Runge-Kutta method of Haras and Ta'asan used in Figure 10 is

$$
\sigma(\lambda h)=1+\lambda h+\frac{(\lambda h)^{2}}{2}+0.166407(\lambda h)^{3}+0.0409525(\lambda h)^{4}+0.0074510(\lambda h)^{5} .
$$

The optimized temporal operator of Zingg, Lomax, and Jurgens [52, 55] is obtained from (8) with the following coefficients:

$$
\begin{aligned}
& \alpha_{1}=0.168850, \\
& \alpha_{2}=0.197348, \\
& \alpha_{3}=0.250038, \\
& \alpha_{4}=0.333306, \\
& \alpha_{5}=0.5 .
\end{aligned}
$$

The resulting characteristic polynomial is

$$
\begin{aligned}
\sigma(\lambda h) & =1+\lambda h+\frac{(\lambda h)^{2}}{2}+0.16665295(\lambda h)^{3}+0.041669557(\lambda h)^{4} \\
& +0.0082233848(\lambda h)^{5}+0.0013885169(\lambda h)^{6}
\end{aligned}
$$

The scheme of Davis is obtained from (10) with

$$
\begin{aligned}
& a_{0}=b_{0}=-2(C-2)(C+2), \\
& a_{1}=b_{2}=(C-1)(C-2), \\
& a_{2}=b_{1}=(C+1)(C+2),
\end{aligned}
$$

where $C=a h / \Delta x$ is the Courant number.

\section{REFERENCES}

[1] S. Abarbanel, D. Gottlieb, and J.S. Hesthaven, Well-posed perfectly matched layers for advective acoustics, J. Comput. Phys., 154 (1999), pp. 266-283.

[2] R.M. Alford, K.R. Kelly, And D.M. Boore, Accuracy of finite-difference modelling of the acoustic wave equation, Geophysics, 39 (1974), pp. 834-842.

[3] D.A. Anderson, J.C. Tannehill, and R.H. Pletcher, Computational Fluid Mechanics and Heat Transfer, McGraw-Hill, New York, 1984, p. 73.

[4] J.-P. Berenger, A perfectly matched layer for the absorption of electromagnetic waves, J. Comput. Phys., 114 (1994), pp. 185-200.

[5] A.C. Cangellaris, C.-C. Lin., and K.K. MeI, Point-matched time-domain finite element methods for electromagnetic radiation and scattering, IEEE Trans. Antennas and Propagation, 35 (1987), pp. 1160-1173. 
[6] M.H. Carpenter, D. Gottlieb, and S. Abarbanel, Stable and accurate boundary treatments for compact, high-order finite-difference schemes, Appl. Numer. Math., 12 (1993), pp. 5587.

[7] M.H. Carpenter, D. Gottlieb, and S. Abarbanel, Time-stable boundary conditions for finite-difference schemes solving hyperbolic systems: Methodology and application to highorder compact schemes, J. Comput. Phys., 111 (1994), pp. 220-236.

[8] G. Cohen And P. Joly, Fourth order schemes for the heterogeneous acoustics equation, Comput. Methods Appl. Mech. Engrg., 80 (1990), pp. 397-407.

[9] M.A. Dablain, The application of high-order differencing to the scalar wave equation, Geophysics, 51 (1986), pp. 54-66.

[10] S. DAVIS, Low-dispersion finite difference methods for acoustic waves in a pipe, J. Acoust. Soc. Amer., 90 (1991), pp. 2775-2781.

[11] M. Fusco, FDTD algorithm in curvilinear coordinates, IEEE Trans. Antennas and Propagation, 38 (1990), pp. 78-89.

[12] S.D. GEDNEY, An anisotropic perfectly matched layer absorbing medium for the truncation of FDTD lattices, IEEE Trans. Antennas and Propagation, 44 (1996), pp. 1630-1639.

[13] D. Gottlieb And E. Turkel, Dissipative two-four methods for time-dependent problems, Math. Comp., 30 (1976), pp. 703-723.

[14] B. Gustafsson, The convergence rate for difference approximations to mixed initial boundary value problems, Math. Comp., 29 (1975), pp. 396-406.

[15] Z. HARAS AND S. TA'ASAn, Finite-difference schemes for long-time integration, J. Comput. Phys., 114 (1994), pp. 265-279.

[16] O. HolberG, Computational aspects of the choice of operator and sampling interval for numerical differentiation in large-scale simulation of wave phenomena, Geophysical Prospecting, 35 (1987), pp. 629-655.

[17] F.Q. Hu, M.Y. Hussaini, AND J.L. Manthey, Low-dissipation and low-dispersion Runge-Kutta schemes for computational acoustics, J. Comput. Phys., 124 (1996), pp. 177-191.

[18] H.M. JURGENS AND D.W. ZINGG, Implementation of a high-accuracy finite-difference scheme for linear wave phenomena, Proceedings of the Third International Conference on Spectral and High Order Methods, Houston, TX, Houston J. Math. (1995).

[19] H.M. JuRgens AND D.W. ZINGG, Numerical solution of the time-domain Maxwell equations using high-accuracy finite-difference methods, SIAM J. Sci. Comput., to appear.

[20] T.G. Jurgens, A. Taflove, K.R. Umashankar, and T.G. Moore, Finite-difference timedomain modeling of curved surfaces, IEEE Trans. Antennas and Propagation, 40 (1992), pp. 357-366.

[21] J.W. Kim And D.J. LeE, Optimized compact finite difference schemes with maximum resolution, AIAA J., 34 (1996), pp. 887-893.

[22] J.D. Lambert, Computational Methods in Ordinary Differential Equations, Wiley, New York, 1973.

[23] S.K. LeLE, Compact finite difference schemes with spectral-like resolution, J. Comput. Phys., 103 (1992), pp. 16-42.

[24] J. Lighthill, The final panel discussion, in Computational Aeroacoustics, J.C. Hardin and M.Y. Hussaini, eds., Springer-Verlag, New York, 1993, pp. 499-513.

[25] Y. Liu, A generalized finite-volume algorithm for solving the Maxwell equations on arbitrary grids, Conference Proceedings of the 10th Annual Review of Progress in Applied Computational Electromagnetics, 1994, pp. 487-494.

[26] Y. LiU, Fourier analysis of numerical algorithms for the Maxwell equations, J. Comput. Phys., 124 (1996), pp. 396-416.

[27] D.P. Lockard, K.S. Brentner, And H.L. Atkins, High accuracy algorithms for computational aeroacoustics, AIAA J., 33 (1994), pp. 246-251.

[28] N.K. MAdSEn AND R.W. Ziolowski, A three-dimensional modified finite volume technique for Maxwell's equations, Electromagnetics, 10 (1990), pp. 147-161.

[29] K.J. Marfurt, Accuracy of finite-difference and finite-element modeling of the scalar and elastic wave equations, Geophysics, 49 (1984), pp. 533-549.

[30] P. Olsson, Summation by parts, projections, and stability I, Math. Comp., 64 (1995), pp. 10351065.

[31] P.G. Petropoulos, Phase error control for FD-TD methods of second and fourth order accuracy, IEEE Trans. Antennas and Propagation, 42 (1994), pp. 859-862.

[32] P.G. Petropoulos, L. Zhao, and A.C. Cangellaris, A reflectionless sponge layer absorbing boundary condition for the solution of Maxwell's equations with high-order staggered finite difference schemes, J. Comput. Phys., 139 (1998), pp. 184-208. 
[33] P.L. RoE, Linear bicharacteristic schemes without dissipation, SIAM J. Sci. Comput., 19 (1998), pp. 1405-1427.

[34] A. SEI, A family of numerical schemes for the computation of elastic waves, SIAM J. Sci. Comput., 16 (1995), pp. 898-916.

[35] P. Sguazzero, M. Kindelan, and A. Kamel, Dispersion-bounded numerical integration of the elastodynamic equations with cost-effective staggered schemes, Comput. Methods Appl. Mech. Engrg., 80 (1990), pp. 165-172.

[36] J.S. Shang, A fractional-step method for the time domain Maxwell equations, J. Comput. Phys., 118 (1995), pp. 109-119.

[37] J.S. Shang And D. Gaitonde, On High Resolution Schemes for Time-Dependent Maxwell Equations, AIAA Paper 96-0832, 1996.

[38] V. Shankar, A.H. Mohammadian, And W.F. Hall, A time-domain finite-volume treatment for the Maxwell equations, Electromagnetics, 10 (1990), pp. 127-145.

[39] G.R. Shubin And J.B. Bell, A modified equation approach to constructing fourth order methods for acoustic wave propagation, SIAM J. Sci. Statist. Comput., 8 (1987), pp. 135-151.

[40] A. Taflove, Computational Electrodynamics: The Finite-Difference Time-Domain Method, Artech House, Boston, 1995.

[41] A. TAFlove, Application of the finite difference time-domain method to sinusoidal steadystate electromagnetic penetration problems, IEEE Trans. Electromagnetic Compatibility, 22 (1980), pp. 191-202.

[42] C.K.W. TAM, Computational aeroacoustics: Issues and methods, AIAA J., 33 (1995), pp. 17881796.

[43] C.K.W. Tam and J.C. WebB, Dispersion-relation-preserving finite difference schemes for computational acoustics, J. Comput. Phys., 107 (1993), pp. 262-281.

[44] E. Turkel And A. Yefet, Fourth order method for Maxwell equations on a staggered mesh, IEEE Antennas and Propagation Society International Symposium 1997 Digest, 4 (1997), pp. 2156-2159.

[45] R. Vichnevetsky and F. De Schutter, A Frequency Analysis of Finite Difference and FiniteElement Methods for Initial Value Problems, in Advances in Computer Methods for Partial Differential Equations, R. Vichnevetsky, ed., AICA/IMACS, Rutgers University, New Brunswick, NJ, 1975, pp. 46-52.

[46] R. Vichnevetsky and J.B. Bowles, Fourier Analysis of Numerical Approximations of Hyperbolic Equations, SIAM Stud. Appl. Math. 5, SIAM, Philadelphia, 1982.

[47] M. Vinokur And M. Yarrow, Finite-Surface Method for the Maxwell Equations in Generalized Coordinates, AIAA Paper 93-0463, 1993.

[48] M. Vinokur and M. Yarrow, Finite-Surface Method for the Maxwell Equations with Corner Singularities, AIAA Paper 94-0233, 1994.

[49] V.L. Wells and R.A. Renaut, Computing aerodynamically generated noise, Annu. Rev. Fluid Mech., 29 (1997), pp. 161-199.

[50] K.S. YEE, Numerical solution of initial boundary value problems involving Maxwell's equations in isotropic media, IEEE Trans. Antennas and Propagation, 14 (1966), pp. 302-307.

[51] J.L. Young, D. Gaitonde, And J.S. Shang, Toward the construction of a fourth-order difference scheme for transient EM wave simulation: Staggered grid approach, IEEE Trans. Antennas and Propagation, 45 (1997), pp. 1573-1580.

[52] D.W. ZingG, H. Lomax, And H.M. Jurgens, An Optimized Finite-Difference Scheme for Wave Propagation Problems, AIAA Paper 93-0459, 1993.

[53] D.W. ZingG AND H. Lomax, Finite-difference schemes on regular triangular grids, J. Comput. Phys., 108 (1993), pp. 306-313.

[54] D.W. ZingG AND H. Lomax, On the eigensystems associated with numerical boundary schemes for hyperbolic equations, in Numerical Methods for Fluid Dynamics, M.J. Baines and K.W. Morton, eds., Clarendon Press, Oxford, UK, 1993, pp. 471-480.

[55] D.W. ZingG, H. Lomax, AND H.M. Jurgens, High-accuracy finite-difference schemes for linear wave propagation, SIAM J. Sci. Comput., 17 (1996), pp. 328-346.

[56] D.W. ZingG, AND T.T. Chisholm, Runge-Kutta methods for linear ordinary differential equations, Appl. Numer. Math., 31 (1999), pp. 227-238.

[57] R.W. Ziolkowski, Time-derivative Lorentz-material model based absorbing boundary conditions, IEEE Trans. Antennas and Propagation, 45 (1997), pp. 1530-1535. 